\title{
CCAR1 5' UTR as a natural miRancer of miR-1254 overrides tamoxifen resistance
}

Gaopeng $\mathrm{Li}^{1,{ }^{*}}$, Xiaoli $\mathrm{Wu}^{1, *}$, Wenchang Qian ${ }^{1,2}$, Huayong Cai ${ }^{1}$, Xinbao Sun ${ }^{1}$, Weijie Zhang ${ }^{1,2}$, Sheng Tan $^{1,2}$, Zhengsheng $\mathrm{Wu}^{3}$, Pengxu Qian ${ }^{4}$, Keshuo Ding ${ }^{3}$, Xuefei Lu ${ }^{1,2}$, Xiao Zhang ${ }^{1}$, Hong Yan ${ }^{3}$, Haifeng Song ${ }^{5}$, Shouhong Guang ${ }^{1,2}$, Qingfa $\mathrm{Wu}^{1,2}$, Peter E Lobie ${ }^{6}$, Ge Shan ${ }^{1,2}$, Tao Zhu ${ }^{1,2}$

${ }^{I}$ The CAS Key Laboratory of Innate Immunity and Chronic Disease, School of Life Sciences, University of Science and Technology of China, Hefei, Anhui 230027, China; ${ }^{2}$ Hefei National Laboratory for Physical Sciences at Microscale, Hefei, Anhui 230027, China; ${ }^{3}$ Department of Pathology, Anhui Medical University, Hefei, Anhui 230027, China; ${ }^{4}$ Stowers Institute for Medical Research, Kansas City, Missouri 64110, USA; ${ }^{5}$ Department of Pharmacology and Toxicology, Beijing Institute of Radiation Medicine, Beijing 100850, China; ${ }^{6}$ Cancer Science Institute of Singapore and Department of Pharmacology, National University of Singapore, Singapore 117599, Singapore

MicroRNAs (miRNAs) typically bind to unstructured miRNA-binding sites in target RNAs, leading to a mutual repression of expression. Here, we report that miR-1254 interacts with structured elements in cell cycle and apoptosis regulator 1 (CCAR1) 5' untranslated region (UTR) and this interaction enhances the stability of both molecules. miR-1254 can also act as a repressor when binding to unstructured sites in its targets. Interestingly, structured miR-1254-targeting sites act as both a functional RNA motif-sensing unit, and an independent RNA functional unit that enhances miR-1254 expression. Artificially designed miRNA enhancers, termed "miRancers", can stabilize and enhance the activity of miRNAs of interest. We further demonstrate that CCAR1 $5^{\prime}$ UTR as a natural miRancer of endogenous miR-1254 re-sensitizes tamoxifen-resistant breast cancer cells to tamoxifen. Thus, our study presents a novel model of miRNA function, wherein highly structured miRancer-like motif-containing RNA fragments or miRancer molecules specifically interact with miRNAs, leading to reciprocal stabilization.

Keywords: CCAR1 5' UTR; miR-1254; miRancer; tamoxifen

Cell Research (2016) 26:655-673. doi:10.1038/cr.2016.32; published online 22 March 2016

\section{Introduction}

MicroRNAs (miRNAs) are small non-coding RNAs that modulate myriad biological processes including cancer progression by repressing the expression of RNA targets. miRNA:mRNA targeting is mainly mediated by specific base-pairing interaction between the $5^{\prime}$ end of miRNA (seed region) and miRNA response elements within the coding region or untranslated regions (UTRs) of mRNA, leading to mRNA destabilization and/or translational inhibition [1-3]. Typically target RNA in return

*These two authors contributed equally to this work.

Correspondence: Tao Zhu

Tel/Fax: +86 55163601505

E-mail: zhut@ustc.edu.cn

Received 16 January 2016; revised 3 February 2016; accepted 3 February

2016; published online 22 March 2016 titrates or sequesters the endogenous miRNA [4]. Conserved seed pairing in unstructured and AU-rich regions, generally located on $3^{\prime}$ UTR, has been considered as a good indicator of potential miRNA target sites [5]. In comparison, the 5' UTR is typically GC-rich and is predicted to possess a higher degree of secondary structure [6]. Although some miRNAs can bind to the 5' UTR of target mRNAs to stabilize the target mRNA [7] or activate transcription [8] or translation [9], the interaction between miRNAs and target RNAs with structured target sites remains largely unexplored.

The selective estrogen receptor modulator, tamoxifen, represents one of the most effective adjuvant treatments for ER $\alpha$-positive $\left(\mathrm{ER}^{+}\right)$breast cancer patients in clinical practice. Unfortunately, many patients develop drug resistance during the treatment. The mechanisms of tamoxifen resistance include aberrant $\mathrm{ER} \alpha$ expression, altered signal transduction pathways (e.g., EGFR, HER2/Neu, 
IGF-1R), changes in miRNA expression, imbalance of co-regulatory proteins (e.g., NCOA1/SRC-1, NCOA2/ GRIP1, NOCA3/AIB1/SRC-3), and genetic polymorphisms in tamoxifen metabolism $[10,11]$. In this study, through screening of miRNAs involved in primary breast cancer, we identified a frequent loss of miR-1254 and its host gene CCARl (cell cycle and apoptosis regulator 1). Study of the interaction between miR-1254 and the 5' UTR of CCAR1 revealed a fascinating paradigm of reciprocal modulation between this miRNA and its highly structured and GC-rich target sites.

\section{Results}

\section{Loss of miR-1254 and CCAR1 expression}

To screen for the potentially deregulated miRNAs at chromosome 10q, a site of frequent loss of heterozygosity in breast cancer [12], we determined the DNA copy numbers of 45 known miRNA genes in this region based on the miRBase 21.0 database [13] in 20 archived breast cancer specimens and 4 specimens from benign breast diseases (Figure 1A and 1B). The frequencies of copy losses or gains were analyzed by genomic quantitative PCR (qPCR) and summarized (Figure 1C). Due to the close proximity of some genes, such as miR-4679-1/2, miR-3158-1/2, miR-6715a/b, which are difficult to distinguish, only 42 items are listed. miR-1254-1 was identified as the most frequently lost (85\%) miRNA gene.

miR-1254-1 resides within the 15th intron of its host gene CCAR1 on 10q21.3 (Figure 1D). CCAR1, also known as CARP1, which has been described as an apoptosis inducer or transcriptional coactivator for nuclear receptors or p53, plays a broad regulatory role in cancer cell progression $[14,15]$. miR-1254-1 and CCAR1 were predicted to share the same transcription start site by Eponine-TSS [16] and miRstart database [17]. The expression of another putative intergenic genome locus of miR-1254 (predicted by miRBase), known as miR-12542 , was below the detection level in breast cancer tissues. As the expression levels of mature pri-miR-1254-1 and miR-1254 are highly concordant (Pearson coefficient $=$ 0.84 , Figure $1 \mathrm{E}$ ), we conclude that miR-1254 is derived mostly, if not entirely, from the miR-1254-1 locus. qRTPCR analysis revealed the reduced expression of primiR-1254-1, miR-1254 and CCAR1 in breast cancer tissues, and their expression levels were highly correlated with each other (Figure 1E-1J). The expression of CCAR1 and mature miR-1254 was further examined in 13 breast epithelial or breast cancer cell lines by qRTPCR. Much higher expression levels of CCAR1 and miR-1254 were observed in 3 immortalized but otherwise normal mammary epithelial cell lines, compared with mammary carcinoma cell lines (Figure $1 \mathrm{~K}-1 \mathrm{~L}$ ). Again, a strong correlation between the expression level of miR-1254 and CCAR1 (Pearson coefficient $=0.71$ ) was observed in these cell lines.

We next sought to functionally establish the legitimacy of miR-1254 as a miRNA. We transfected MCF-7 and T-47D cells with pri-miR-1254 expression plasmid containing miR-1254 precursor with $300 \mathrm{nt}$ flanking sequence at either side (Supplementary information, Figure S1A) and performed northern blot analysis, which is considered as the gold standard for verifying a candidate miRNA, using digoxin-tagged DNA oligos antisense to either miR1254 or let-7a. The endogenous pre-miRNAs as well as mature miRNAs were detected by their respective specific probes. Increased expression of miR-1254 but not let$7 \mathrm{a}$ was detected as a result of forced expression of primiR-1254-1 (Supplementary information, Figure S1B and S1C). In addition, the expression of miR-1254 is dependent on the expression of Drosha, Dicer and Ago2, as the expression was abrogated in the absence of these proteins caused by shRNA (Supplementary information, Figure S1D). Thus, miR-1254 is an authentic miRNA.

The extraordinarily low reads of miR-1254 by RNASeq in miRbase was most probably due to a very high GC content $(62.5 \%)$ in miR-1254 (Supplementary information, Figure S1E). We found that reverse transcription using superscript III at $50{ }^{\circ} \mathrm{C}$ or $55^{\circ} \mathrm{C}$ was required for the detection of miR-1254 by qPCR (Supplementary information, Figure $\mathrm{S} 1 \mathrm{G}$ and $\mathrm{S} 1 \mathrm{H}$ ), whereas miR-1254 was minimally detectable using superscript II for reverse transcription at $42^{\circ} \mathrm{C}$ or $44^{\circ} \mathrm{C}$ as used in the preparation of small RNA library by different groups in miRBase (Supplementary information, Figure S1F). As control, miR-7 (GC 34.8\%) or miR-9 (GC 34.8\%) could be efficiently reverse-transcribed under all of above conditions (Supplementary information, Figure S1G).

\section{Reciprocal stabilization of miR-1254 and CCARI}

As intronic miRNAs have been reported to influence the expression of their host genes via transcriptional regulation [8], we investigated possible functional interaction between CCARl and miR-1254 in light of their highly correlated expression. We first tested whether miR-1254 could promote CCAR1 transcription. A 1.14 $\mathrm{kb}$ fragment containing CCAR1 promoter (1 kb upstream of start codon) and exon 1 was inserted into pGL3-basic vector upstream of firefly luciferase gene, termed as $\mathrm{CPE}$ (Supplementary information, Figure S2C), which was co-transfected with pRL-TK vector to allow normalization with Renilla luciferase activity. miR-1254 did not affect the promoter activity of CCAR1 (Supplementary information, Figure S2G). Further, forced expression of 
A

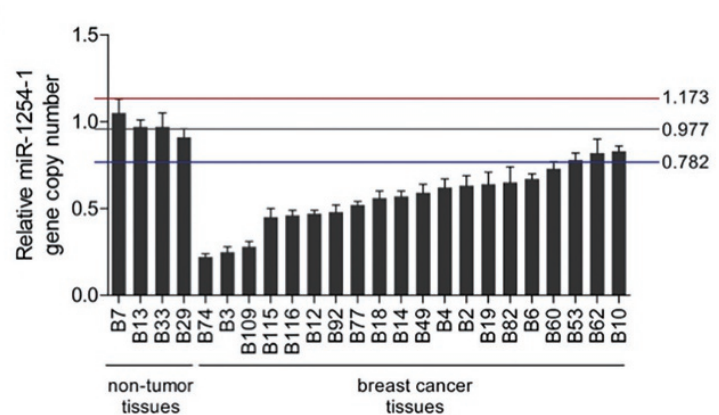

B

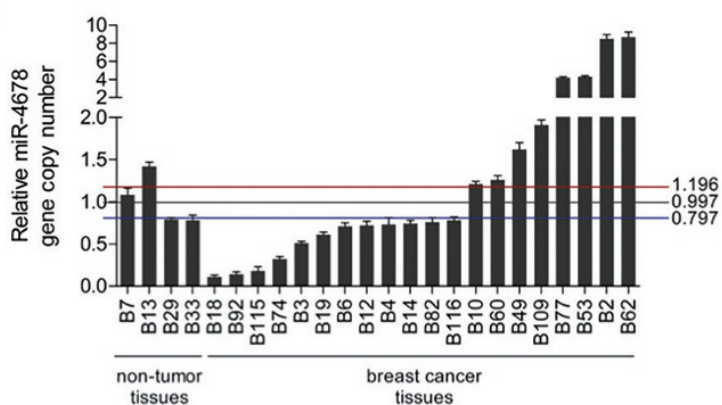

C

\begin{tabular}{|c|c|c|c|c|}
\hline No. & ID & $\begin{array}{l}\text { Genome } \\
\text { Location }\end{array}$ & $\begin{array}{l}\text { Deletion } \\
\text { Frequency }\end{array}$ & $\begin{array}{c}\text { Amplification } \\
\text { Frequency }\end{array}$ \\
\hline 1 & miR- 5100 & $10 \mathrm{q} 11.21$ & $60 \%$ & 0 \\
\hline 2 & miR- 3156-1 & $10 \mathrm{q} 11.21$ & $70 \%$ & 0 \\
\hline 3 & miR- 4294 & $10 q 11.23$ & $65 \%$ & $5 \%$ \\
\hline 4 & miR- 605 & $10 q 21.1$ & $80 \%$ & $10 \%$ \\
\hline 5 & miR- $548 f-1$ & $10 q 21.1$ & $65 \%$ & $30 \%$ \\
\hline 6 & miR- 3924 & $10 q 21.1$ & $65 \%$ & 0 \\
\hline 7 & miR- 1296 & $10 q 21.3$ & $70 \%$ & 0 \\
\hline 8 & miR-1254-1 & $10 q 21.3$ & $85 \%$ & 0 \\
\hline 9 & miR- 4676 & $10 q 22.1$ & $65 \%$ & 0 \\
\hline 10 & miR- 606 & $10 q 22.2$ & $55 \%$ & $10 \%$ \\
\hline 11 & miR- 346 & $10 q 23.2$ & $65 \%$ & 0 \\
\hline 12 & miR- 4678 & $10 \mathrm{q} 23.2$ & $55 \%$ & $40 \%$ \\
\hline 13 & miR- $4679-1 / 2$ & $10 \mathrm{q} 23.31$ & $45 \%$ & $35 \%$ \\
\hline 14 & miR- 107 & $10 \mathrm{q} 23.31$ & $60 \%$ & $5 \%$ \\
\hline 15 & miR- 3157 & $10 \mathrm{q} 24.1$ & $65 \%$ & 0 \\
\hline 16 & miR- 607 & $10 q 24.1$ & $65 \%$ & 0 \\
\hline 17 & miR- 5686 & $10 \mathrm{q} 24.1$ & $65 \%$ & $5 \%$ \\
\hline 18 & miR- 1287 & $10 q 24.2$ & $70 \%$ & $5 \%$ \\
\hline 19 & miR- 4685 & $10 \mathrm{q} 24.2$ & $55 \%$ & $5 \%$ \\
\hline 20 & miR- 6507 & $10 \mathrm{q} 24.2$ & $60 \%$ & $5 \%$ \\
\hline 21 & miR- 608 & $10 \mathrm{q} 24.31$ & $55 \%$ & 0 \\
\hline 22 & miR- 3158-1/2 & $10 \mathrm{q} 24.32$ & $65 \%$ & 0 \\
\hline 23 & miR-146b & $10 \mathrm{q} 24.32$ & $80 \%$ & 0 \\
\hline 24 & miR- 1307 & $10 q 24.33$ & $70 \%$ & 0 \\
\hline 25 & miR- 936 & $10 \mathrm{q} 25.1$ & $75 \%$ & 0 \\
\hline 26 & miR- 609 & $10 q 25.1$ & $60 \%$ & $5 \%$ \\
\hline 27 & miR- 4482 & $10 q 25.1$ & $60 \%$ & 0 \\
\hline 28 & miR- 4680 & $10 q 25.2$ & $50 \%$ & $15 \%$ \\
\hline 29 & miR- $548 \mathrm{e}$ & $10 q 25.2$ & $55 \%$ & 0 \\
\hline 30 & miR- $6715 a / b$ & $10 q 25.2$ & $60 \%$ & $5 \%$ \\
\hline 31 & miR- 4295 & $10 \mathrm{q} 25.2$ & $60 \%$ & 0 \\
\hline 32 & miR- 4483 & $10 q 25.3$ & $60 \%$ & 0 \\
\hline 33 & miR- 2110 & $10 q 25.3$ & $70 \%$ & $5 \%$ \\
\hline 34 & miR- 3663 & $10 q 25.3$ & $35 \%$ & $5 \%$ \\
\hline 35 & miR- 4681 & $10 \mathrm{q} 26.11$ & $80 \%$ & 0 \\
\hline 36 & miR- 4682 & $10 \mathrm{q} 26.12$ & $65 \%$ & 0 \\
\hline 37 & miR- 3941 & $10 \mathrm{q} 26.13$ & $50 \%$ & $40 \%$ \\
\hline 38 & miR- 4296 & $10 \mathrm{q} 26.13$ & $60 \%$ & $5 \%$ \\
\hline 39 & miR- 4297 & $10 q 26.3$ & $60 \%$ & 0 \\
\hline 40 & miR- 378c & $10 q 26.3$ & $55 \%$ & $5 \%$ \\
\hline 41 & miR- 202 & $10 q 26.3$ & $60 \%$ & $5 \%$ \\
\hline 42 & miR- 3944 & $10 \mathrm{q} 26.3$ & N/A* & $\mathrm{N} / \mathrm{A}^{*}$ \\
\hline
\end{tabular}

*Not available in this system.
D

Chromosome 10

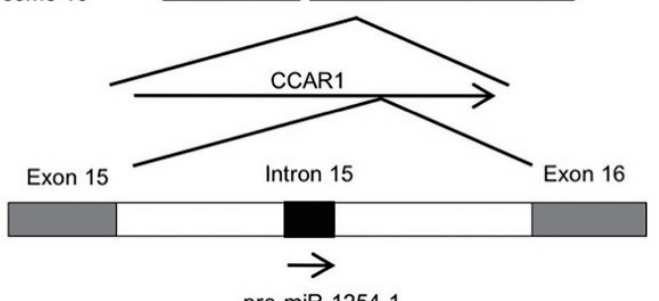

E

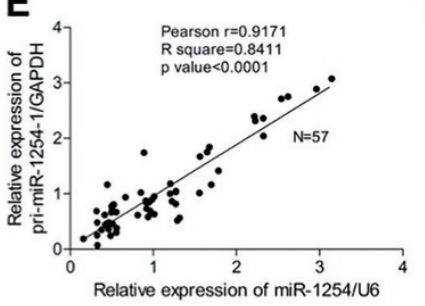

H

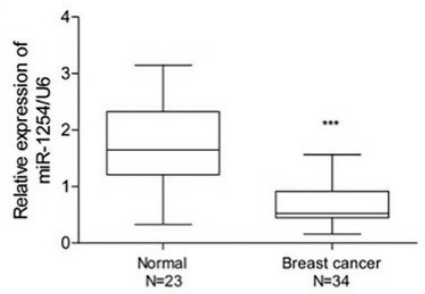

F
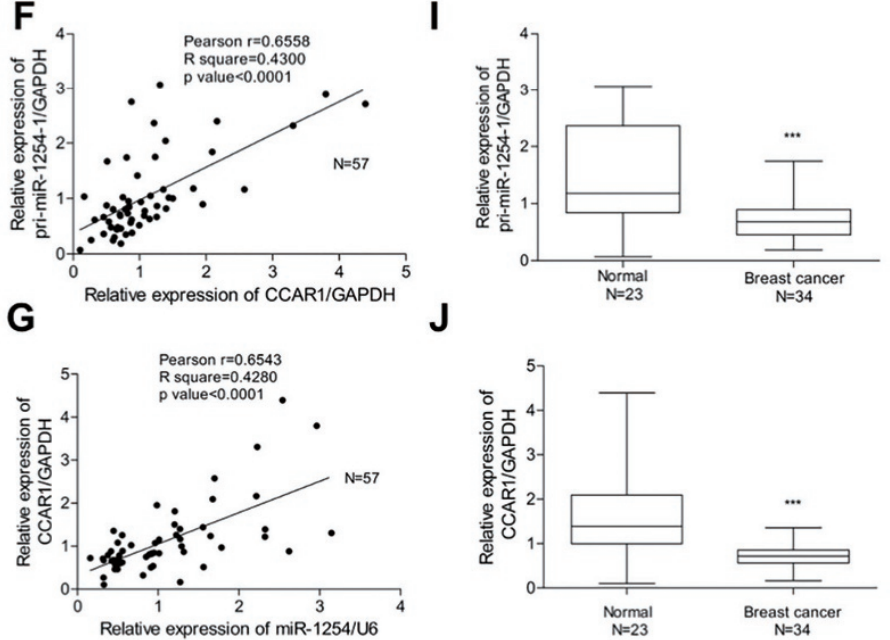

K

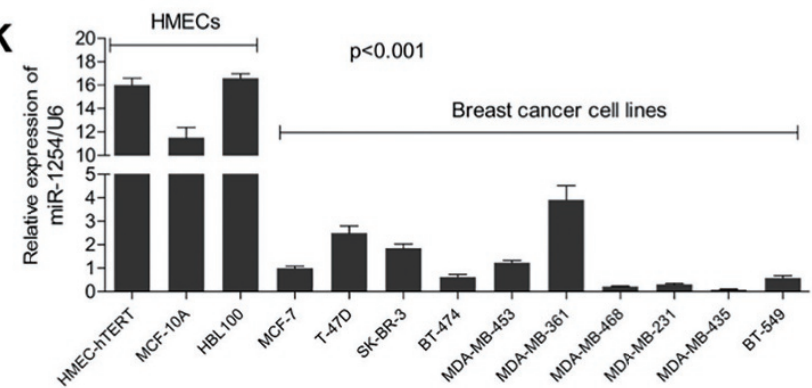

$\mathbf{L}$

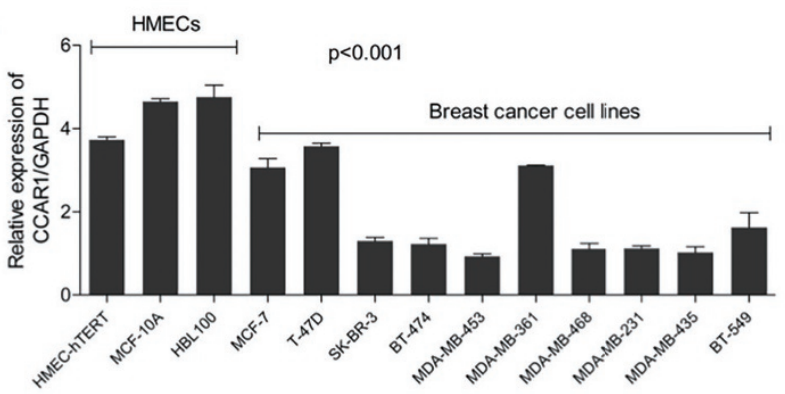


miR-1254 promoted the expression of CCAR1 but not pri-CCAR1 (Figure 2A), whereas miR-1254 antagonism by a miRNA sponge [18] (Supplementary information, Figure S2A, S2B and S2E) decreased the level of CCAR1 but not pri-CCAR1 (Figure 2B) in MCF-7 cells, suggesting that regulation occurs at the posttranscriptional level. Consistently, forced expression of miR-1254 promoted CCAR 1 protein expression, whereas miR-1254 sponge reduced expression of CCAR1 protein (Figure $2 \mathrm{C}$ ). We further found that forced expression of miR1254 extended the half-life of CCAR1 mRNA whereas miR-1254 antagonism accelerated CCAR1 mRNA decay (Figure 2D). Similar effects were observed in another luminal $\mathrm{A} / \mathrm{ER}^{+}$cell line T-47D (Supplementary information, Figure S2F). As both 3' UTR and 5' UTR could be involved in the regulation of mRNA stability [19], we first excluded a role of CCAR1 3' UTR in the regulation of miR-1254-mediatied stabilization of CCAR1 mRNA by using a CCAR1 3' UTR luciferase reporter plasmid (LUC-C3U) (Supplementary information, Figure S2D and S2J). Furthermore, a CCAR1 5' UTR (C5U) reporter plasmid, namely C5U-LUC (Figure 2E), was employed to determine miR-1254 activity on CCAR $15^{\prime}$ UTR. Forced expression of miR-1254 increased C5U-LUC mRNA level (Figure 2F) and C5U-LUC reporter activity (Figure 2G), which were significantly abrogated by miR-1254 sponge (Supplementary information, Figure $\mathrm{S} 2 \mathrm{H}$ and S2I). We further found by mutagenesis that two miR-1254-binding sites calculated by RNA hybrid [20] on $\mathrm{C} 5 \mathrm{U}$, namely site 1 and site 2 (Figure $2 \mathrm{H}$ ), were both required for miR-1254-elicited effects on C5U (Figure $2 \mathrm{~F}$ and $2 \mathrm{G})$.

To determine the possible physical association between C5U and miR-1254, affinity purification of miR-1254-interacting endogenous CCAR 1 mRNA in cytosolic extract was performed (Supplementary information, Figure S2K). Approximately 30-fold enrichment of CCAR1 mRNA was detected by the use of 3'-biotin-tagged miR-1254 (Supplementary information, Figure S2L). The cognate miR-1254 with 6 nt substi- tutions in the seed region and a bantam miRNA, both tagged with biotin, were included as controls. Of note, mutant miR-1254 still conferred a $\sim 7$-fold enrichment of CCAR1 mRNA, suggesting that a non-seed match might partially contribute to their binding (Supplementary information, Figure S2L). As controls, either wild-type or mutant miR-1254 failed to enrich GAPDH mRNA (Supplementary information, Figure S2M). Thus, these data suggest that miR-1254 interacts with CCAR1 5' UTR via a base-pairing mechanism.

To further define the effect of miR-1254 on CCAR1 5 ' UTR, we constructed a recombinant plasmid namely CEG, transcribing a chimeric mRNA containing an EGFP coding sequence flanked by $\mathrm{C} 5 \mathrm{U}$ and GAPDH 3' UTR (G3U) (Figure 2I). Consistently, the expression levels and decay of EGFP were regulated by miR-1254; this effect depended on the two wild-type miR-1254binding sites in C5U (Figure 2J-2L), and was lost when both miR-1254-binding sites in C5U were mutated (mutCEG). A dose-dependent expression of EGFP protein was detected by manipulating miR-1254 levels in MCF-7 CEG cells but not in MCF-7 mutCEG cells (Figure 2M). Together, we conclude that miR-1254 increases CCAR1 expression by enhancing its mRNA stability.

The possible reciprocal modulation of miR-1254 expression by $\mathrm{C} 5 \mathrm{U}$ was examined. Interestingly, $\mathrm{CEG}$ overexpression resulted in a $\sim 5$-fold increase in miR1254 levels, whereas CCAR1 depletion by pooled shRNAs reduced miR-1254 levels by $\sim 70 \%$ (Figure $2 \mathrm{~N}$ and 2O). Notably, the endogenous CCAR1 level was slightly reduced upon forced expression of CEG (Figure $2 \mathrm{~N}$ ), possibly due to competitive absorption of endogenous CCAR1-stabilizing factors by CEG. pri-miR-1254-1 level was unaffected by C5U (Figure $2 \mathrm{~N}$ and 2O), further suggesting C5U-potentiated miR-1254 expression occurs at the post-transcriptional level. Finally we found that the half-life of miR-1254 was significantly elevated upon $\mathrm{C} 5 \mathrm{U}$ overexpression, whereas a much faster decay was observed upon CCAR1 depletion (Figure 2P).

For reciprocal verification, 3'-biotin-tagged probes

Figure 1 Frequent loss of miR-1254 and CCAR1 expression in breast cancer. (A-B) Genomic qPCR of miR-1254-1 (A) and miR-4678 (B) in normal breast tissues or breast cancer tissues; the red lines indicate the threshold for gene amplification and the blue lines indicate the threshold for gene deletion. Error bar indicates standard error of the mean (SEM). ${ }^{* * *} P<0.001$; NS, not significant. (C) List of miRNAs on chromosome 10q frequently deleted or amplified in breast carcinoma determined by genomic qPCR. miR-3944 is below the detection level. (D) Schematic diagram of the genomic locus of hsa-miR-1254-1. (E-G) Correlation between the expression levels of miR-1254 and pri-miR-1254-1 (E), CCAR1 and pri-miR-1254-1 (F) miR1254 and CCAR1 (G) in 57 breast epithelial tissues. Error bars indicate SEM. ${ }^{* * *} P<0.001$. (H-J) miR-1254 (H), pri-miR-1254 (I) and CCAR1 (J) expression in normal breast tissues or breast cancer tissues determined by qRT-PCR. Error bars indicate SEM. ${ }^{* * *} P<0.001$. (K-I) Expression levels of miR-1254 (K) and CCAR1 (L) in 13 human mammary epithelial cell lines (HMECs) analyzed by qRT-PCR. Error bars indicate SEM. ${ }^{* * *} P<0.001$. Student's $t$-test. See also Supplementary information, Figure S1. 
A

E

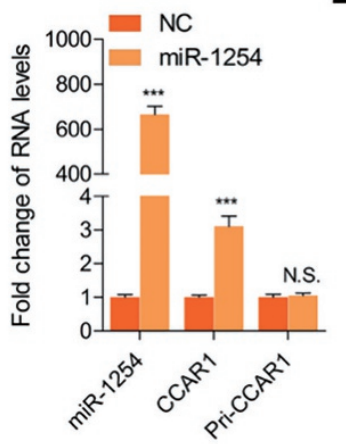

C5U-LUC

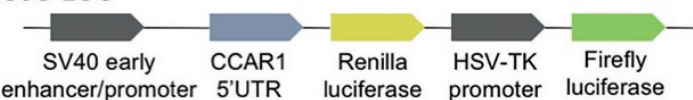

$\mathbf{F}$

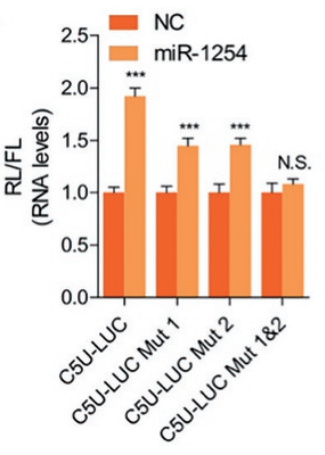

B

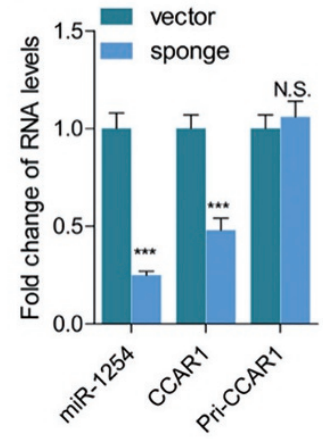

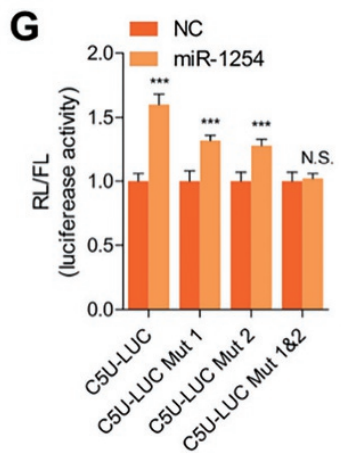

C

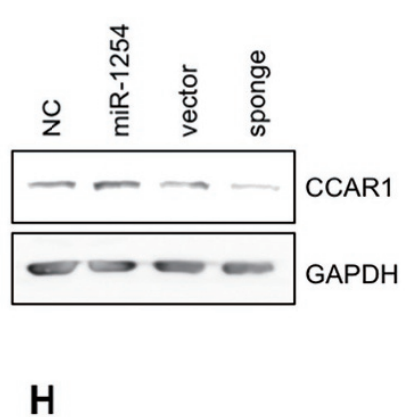

D

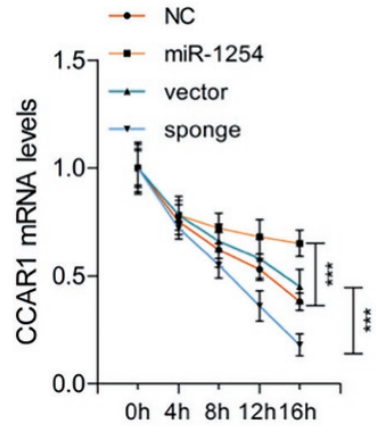

site1 : position 29

hsa-miR-1254 3'- UGACGUCCGAGGUCGA--AGGUCCGA -5'

Human CCAR1 5'- AGUAAGCGCC-GUUCCUCCGGGAG -3'

C5U Mut 1 5'- AgUaAgCGCC-GUUCCUAUAUAag - $3^{\prime}$

site 2 : position 123

hsa-miR-1254 3'- UGAC-GUCCGAG----GUCGAA----------GGUCCGA -5'

Human CCAR1 5' - AGCUGACGGGUUUGAAAUGGCUUCGAUGUUAGCCGGGAC - 3 '

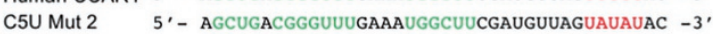

\section{I}
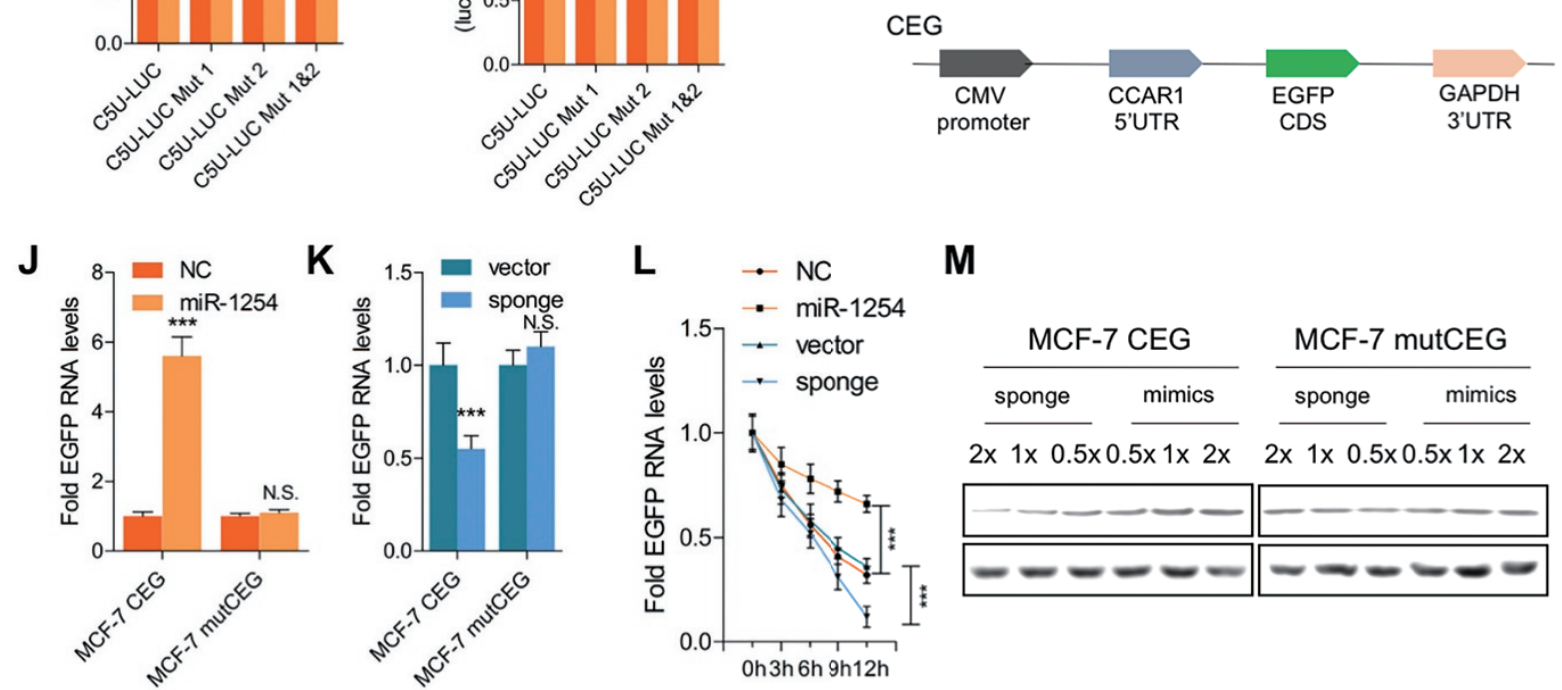

M

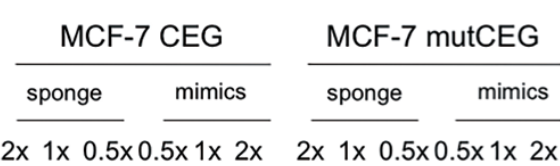

$\mathbf{N}$

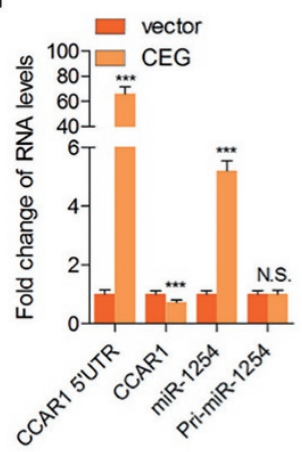

$\mathbf{0}$

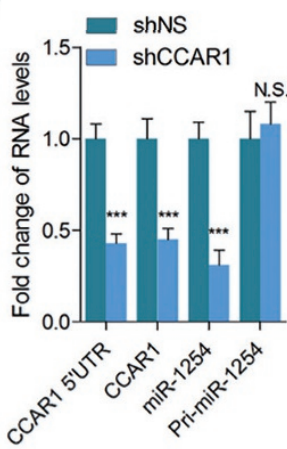

$\mathbf{P}$

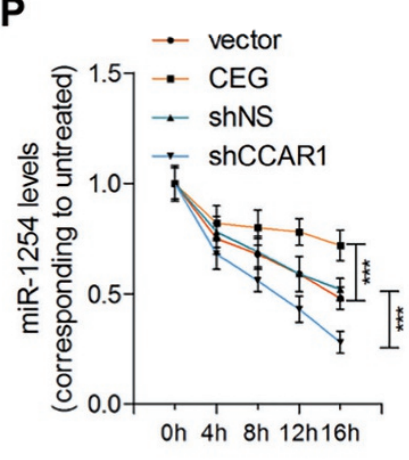


were used to pull down CCAR1 transcript-associated miRNAs. Significant enrichment of miR-1254 was observed with C5U-specific probes but not the 3'-biotin-tagged lacZ probes (Supplementary information, Figure S2N and S2O). In contrast, neither C5U-specific probes nor lacZ-specific probes enriched let-7a (Supplementary information, Figure S2P). Consistently, the enrichment of miR-1254 was further enhanced upon co-transfection of CEG but not mutCEG (Supplementary information, Figure S2O).

\section{Mechanism of miR-1254/CCAR1 interaction}

To gain mechanistic insight into miR-1254/CCAR1 interaction, we first sought to determine whether Argonaute-associated miRISC is involved. Interestingly, depletion of Ago 2 but not Ago1 by pooled specific shRNAs reduced the expression of both CCAR1 and miR-1254 (Figure 3A). The reciprocal potentiation (Figure $3 \mathrm{~B}$ and 3D) and stabilization (Figure $3 \mathrm{C}$ and $3 \mathrm{E}$ ) between miR1254 and CCAR1 were substantially abrogated by Ago2 depletion, suggesting an essential role of Ago2 in miR1254/CCAR1 interaction. We subsequently interrogated if Ago 2 directly interacted with this miRNA/mRNA complex. Interestingly, EGFP mRNA and thereby C5U was only detected in Ago2 immunoprecipitates using cytosolic extract derived from MCF-7 CEG cells transfected with miR-1254 but not negative control (NC, Figure 3F). Reciprocally, miR-1254 was significantly enriched to a greater extent in Ago2 immunoprecipitates using cytosolic extract derived from MCF-7 cells transfected with CEG than that of mutCEG (Figure 3G). Consistently, quantification of biotin-labeled miR-1254-associated CCAR 1 mRNA or C5U-associated miR-1254 indicated that Ago2 depletion substantially diminished the interaction between miR-1254 and CCAR 1 mRNA (Figure 3H and 3I). Hence, miR-1254 binds the CCAR1 5' UTR in association with Ago2.

Because the canonical role of Ago2-associated miRISC is to mediate miRNA-elicited target silencing, we thus explored the mechanism by which Ago2/miRISC facilitates miRNA/target mutual stabilization by examining the structural and sequence features of $\mathrm{C} 5 \mathrm{U}$. Interestingly, as predicted by RNAfold [21], the two miR-1254binding sites in $\mathrm{C} 5 \mathrm{U}$ exhibited similar hairpin-like RNA structures (Figure 3J). In addition, the predicted secondary RNA structures of miR-1254-binding sites, either as an integral part of the $\mathrm{C} 5 \mathrm{U}$ or as isolated RNA fragments, are exactly the same, suggesting the folding of miR1254-binding regions is independent of the flanking RNA sequences. These two RNA fragments were subsequently subcloned into pSilencer 4.1 CMV plasmid, wherein the insertion sequence was juxtaposed to the CMV transcription start site and followed by a synthetic minimal polyA cassette. Interestingly, expression of either of the isolated structured miR-1254-binding site 1 or 2 (namely SS1 or SS2) was sufficient to partially recapitulate the capacity of $\mathrm{C} 5 \mathrm{U}$ to enhance the expression and activity of miR1254 (Figure 3L and 3M).

More strikingly, use of the linear sites (namely LS1 or LS2), which were derived by disrupting the hairpin structure of SS1 or SS2 without affecting their miR-1254binding sequences (Figure $3 \mathrm{~K}$ ) converted the miRNA-enhancing molecules (SS1 or SS2) into miRNA-titrating molecules, leading to reduced expression (Figure 3L) and activity (Figure 3M) of miR-1254. The luciferase reporter plasmids containing SS1/SS2 or LS1/LS2 in front of the luciferase gene were further constructed (Figure $3 \mathrm{~N})$. miR-1254 overexpression significantly increased the luciferase activity and mRNA levels in cells transfected with the reporter plasmid containing SS1 or SS2

Figure 2 Reciprocal stabilization of miR-1254 and CCAR1. (A-B) MCF-7 cells were transfected with negative control (NC), miR-1254 mimic (miR-1254), pcDNA3.1 (+) (vector) or miR-1254 sponge (sponge). The levels of miR-1254, CCAR1 and priCCAR1 (primary CCAR1 transcript) were determined by qPCR. Error bars indicate SEM. ${ }^{* * *} P<0.001$, NS, not significant. (C) Western blot analysis of CCAR1 with forced expression or antagonism of miR-1254. (D) Half-life of CCAR1 mRNA determined after forced expression or repression of miR-1254 in the presence of actinomycin D. Error bars indicate SEM. ${ }^{* * *} P<$ 0.001. (E) Schematic diagram of the C5U-LUC plasmid. (F-G) MCF-7 cells were co-transfected with miR-1254 and C5U-LUC or a single-site mutant (C5U-LUC-Mut 1, C5U-LUC-Mut 2) or a double-site mutant (C5U-LUC-Mut 1 and 2), and luciferase mRNA level $(\mathbf{F})$ or luciferase activity $(\mathbf{G})$ of $\mathrm{FL} / \mathrm{RL}$ was determined. Error bars indicate SEM. ${ }^{* * *} P<0.001$. (H) Alignment of miR-1254 and its binding sequences on C5U. (I) Schematic diagram of CEG plasmid. (J-K) EGFP RNA levels of MCF-7 CEG and MCF-7 mutCEG cells upon forced expression (J) or repression (K) of miR-1254. Error bars indicate SEM. ${ }^{* * *} P<0.001$. (L) Half-life of EGFP mRNA determined in MCF-7 CEG cells upon forced expression or repression of miR-1254 in the presence of actinomycin D. Error bars indicate SEM. ${ }^{* * *} P<0.001$. (M) Protein levels of EGFP and $\beta$-ACTIN were determined after transfection with different doses of miR-1254 mimic or sponge in MCF-7 CEG and MCF-7 mutCEG cells. (N-O) MCF-7 cells transfected with vector or CEG (N), or shRNA of non-specific sequence (shNS) or shCCAR1 (0) for 48 h; the levels of CCAR1 5' UTR, CCAR1, miR-1254, and pri-miR-1254 (primary miR-1254 transcript) were determined by qPCR. Error bars indicate SEM. ${ }^{* * *} P<0.001$. (P) Half-life of miR-1254 upon forced expression or depletion of C5U in presence of actinomycin D. Error bars indicate SEM. ${ }^{* * *} P<0.001$. Student's $t$-test. See also Supplementary information, Figure S2. 

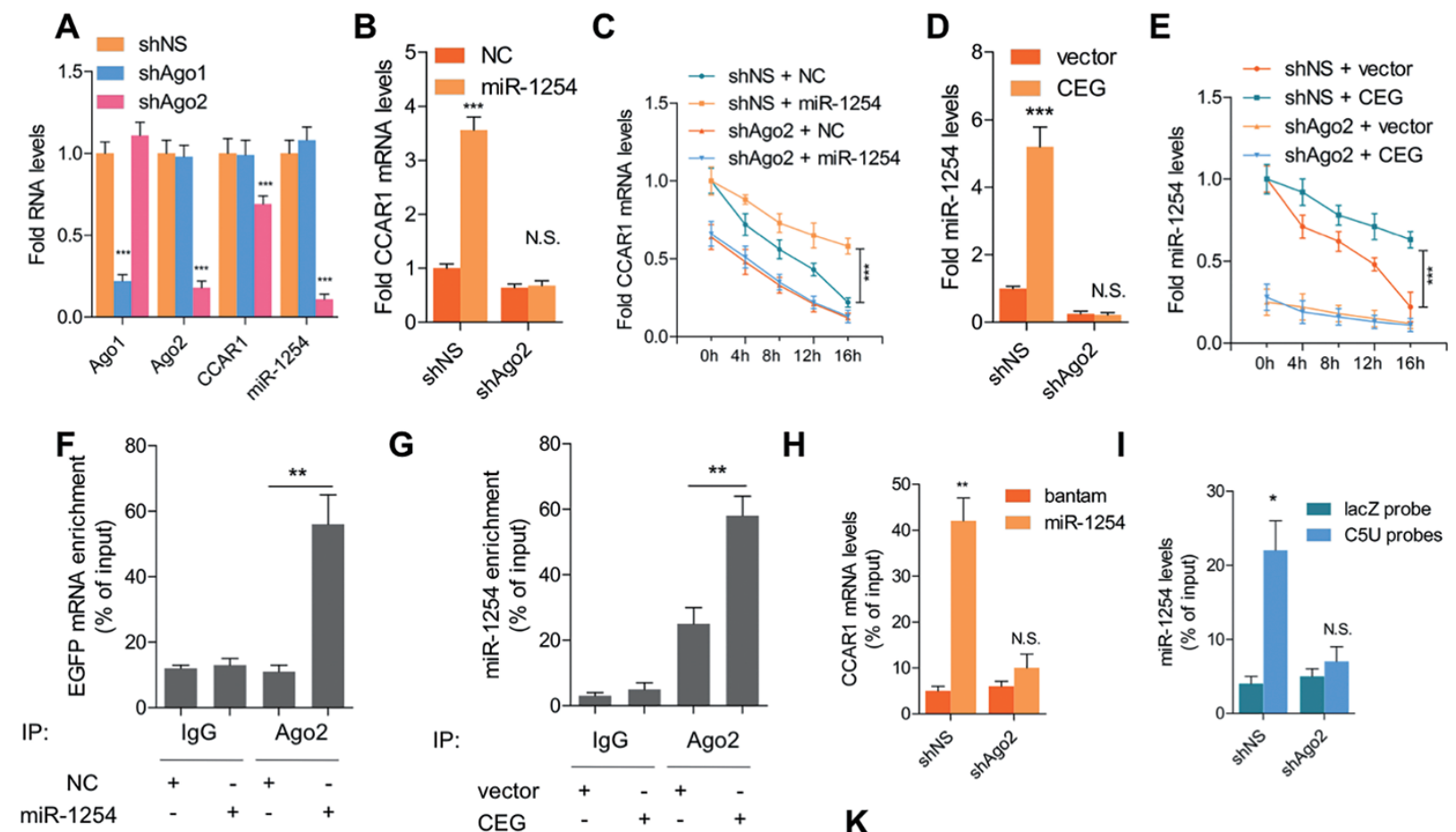

G

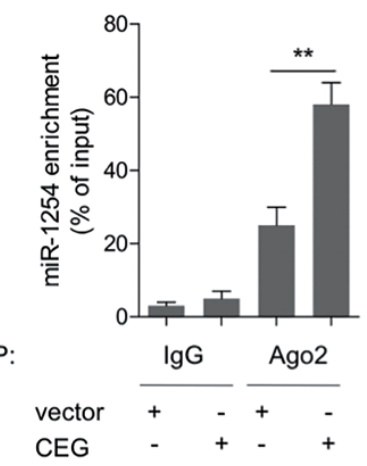

H
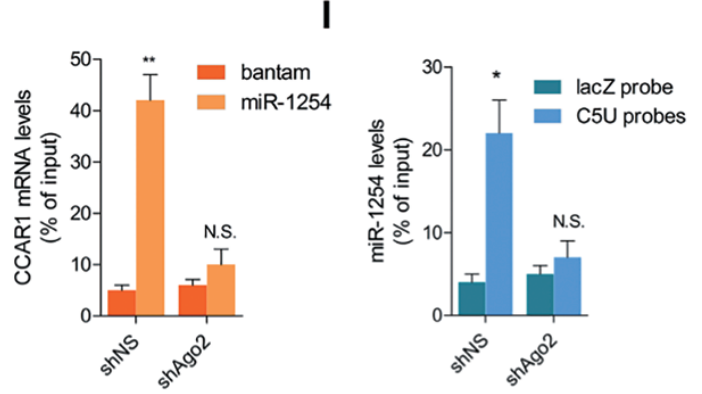

IB: Ago2

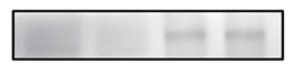

IB: Ago2

$\mathbf{K}$

$$
\begin{aligned}
& \text { miR-1254 3'- UGACGUCCGAGGUCGA--AGGUCCGA -5' }
\end{aligned}
$$

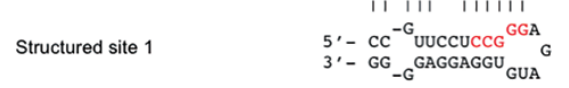

$$
\begin{aligned}
& \text { Linear site } 1 \text { 5'- CCGUUCCUCCGGGag -3' }
\end{aligned}
$$
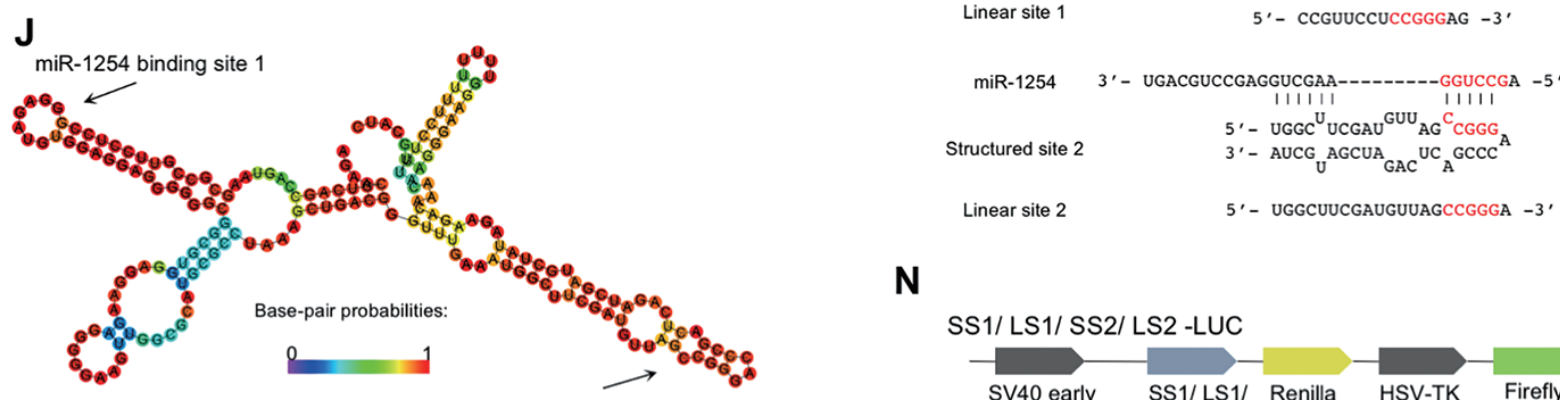

$\mathbf{N}$

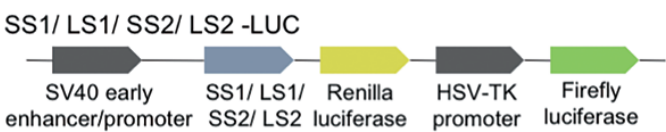

$\mathbf{L}$

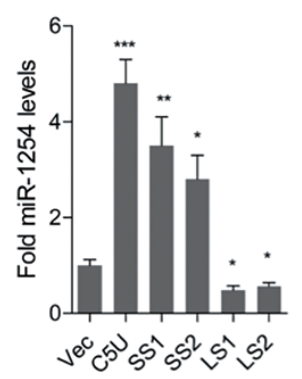

M

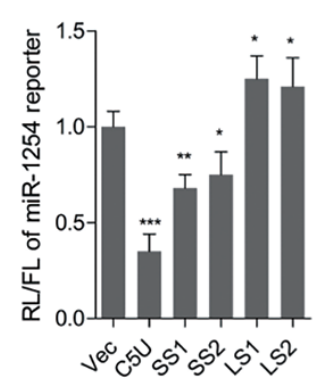

o

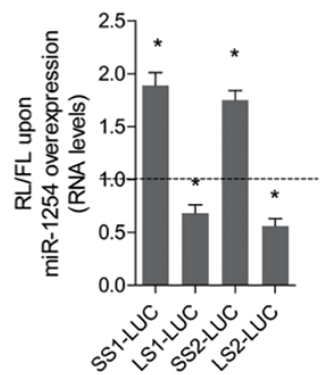

$\mathbf{P}$

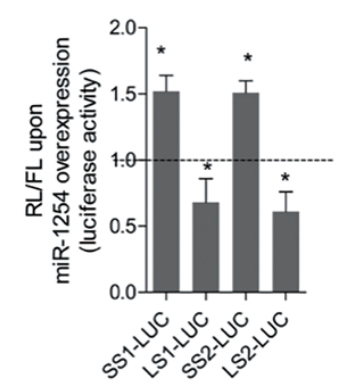


but repressed the luciferase activity and mRNA levels in cells transfected with the reporter plasmid containing LS1 or LS2 (Figure $3 \mathrm{O}$ and 3P). These data suggest that the hairpin RNA structure of the miR-1254-binding sites is also critical for the mutual stabilization of miR-1254 and its structured targets.

To extend the miR-1254/CCAR1 interaction paradigm to other RNA transcripts, we made use of Ago2-PARCLIP data set derived from MCF-7 cells to screen for $5^{\prime}$ UTRs containing potential structured miR-1254-binding sites genome-wide. The RNA fragments containing at least five reads and one $\mathrm{T}$ to $\mathrm{C}$ conversion of a unique cluster were considered as AGO2-binding sites [22]. The clusters which overlap with miR-1254 binding sites-containing $5^{\prime}$ UTRs, featured by 6-7 mer perfect match with the seed region of miR-1254 (allowing G-U pairs), were subsequently retrieved. As a result, we found 9 Ago2-interacting transcripts, the 5' UTRs of which contain putative miR-1254-binding sites (Supplementary information, Figure S3A). TP53INP1 was further identified as the only gene with elevated expression by qRT-PCR upon miR-1254 overexpression (Supplementary information, Figure S3B). Fascinatingly, the predicted three miR-1254-binding sites on TP53INP1 5' UTR (T5U) all presented hairpin-like structures as predicted by RNAhybrid and RNAfold, which mimic the structural and sequence features of miR-1254-binding sites on C5U (Supplementary information, Figure S3C). Reporter plasmids containing T5U or each of the individual structured miR1254-binding site (SS1/SS2/SS3) or unstructured miR1254-binding site (LS1/LS2/LS3) derived from T5U were further constructed (Supplementary information, Figure S3D). We observed that miR-1254 overexpression increased the reporter activity and luciferase mRNA lev- el in cells transfected with reporter plasmids containing T5U or any individual structured miR-1254-binding site, whereas the reporter activity and luciferase mRNA level were reduced in cells transfected with reporter plasmids containing any individual linear miR-1254-binding site upon miR-1254 overexpression (Supplementary information, Figure S3E and S3F). These findings suggest miR1254 could specifically elevate the expression of genes with structured miR-1254-binding sites in their 5' UTRs.

\section{Rational design of miRancer}

Extracting from the structural and sequence similarity shared by two miR-1254-binding sites, RNA fragments mimicking miR-1254-binding RNA sites termed "miRNA enhancers" or "miRancers", which possess a shortened or simplified sequence but retain a similar RNA structure compared with the prototypes, were artificially designed and expressed in pSilencer 4.1 CMV (Figure 4A). miRancer-1254 significantly stabilized miR 1254 and further increased the expression and activity of miR-1254, while mutation in the miR-1254 seed region binding sequence in miRancer-1254 abrogated its efficacy (Figure 4B to 4D). Consistently, unstructural miRancer containing linear miRNA-binding site (MBS) resulted in its conversion into a miRNA titrator or sponge (Figure 4B to 4D). Further rational design of miRancers for miR-7 (miRancer-7) and miR-9 (miRancer-9), was performed (Figure 4E). We employed the luciferase reporter plasmid containing the 3' UTR of PTK2 or CDH1 to measure the activity of miR-7 or miR-9, respectively [23, 24]. miRancer-7 or miRancer-9 specifically enhanced the expression (Figure $4 \mathrm{~F}$ ) and activity (Figure 4G) of miR-7 or miR-9, respectively, whereas linear MBS (miR-7 sponge or miR-9 sponge) specifically reduced the expression (Figure $4 \mathrm{H}$ )

Figure 3 Mechanism of miR-1254 and CCAR1 interaction in association with Ago2. (A) MCF-7 cells were transfected with shRNA of non-specific sequence (shNS), pooled shRNAs for Ago1 (shAgo1) or Ago2 (shAgo2). RNA levels of Ago1, Ago2, miR1254 and CCAR1 were determined by qPCR. Error bars indicate SEM. ${ }^{* * *} P<0.001$. (B-C) Ago2 depletion abrogates miR1254's effect in promoting the expression (B) and half-life (C) of CCAR1. Error bars indicate SEM. ${ }^{* * *} P<0.001$. (D-E) Ago2 depletion abrogates CCAR1 5' UTR-mediated increased expression (D) and half-life (E) of miR-1254. Error bars indicate SEM. ${ }^{* * *} P<0.001$. (F-G) RNA immunoprecipitation of Ago2 or rabbit IgG in MCF-7 CEG cells with forced expression of miR-1254 $(F)$, or in MCF-7 cells with forced expression of CCAR1 5' UTR (G). EGFP and miR-1254 were detected by qRT-PCR and measured with input. Error bars indicate SEM. ${ }^{* *} P<0.01$. (H-I) Ago2 depletion abrogates CCAR1 enrichment by biotin-tagged miR-1254 (H) and miR-1254 enrichment by biotin-tagged probes against CCAR1 5' UTR (I). Error bars indicate SEM. * $P<0.05$, ${ }^{* *} P<0.01$. (J) Secondary structure of CCAR1 5' UTR predicted by RNA fold. miR-1254-binding sites are indicated by arrows. (K) Sequence alignment of miR-1254 and structured site1 (SS1), linear site 1 (LS1), structured site 2 (SS2) and linear site 2 (LS2). (L-M) miR-1254 levels (L) or RL/FL of miR-1254 reporter (M) were determined upon transfection with pSilencer vector (Vec) or expression plasmids for CCAR1 5' UTR (C5U), SS1, LS1, SS2, LS2. Error bars indicate SEM. ${ }^{*} P<0.05,{ }^{* *} P<0.01,{ }^{* * *} P<0.001$. (N) Schematic diagram of the SS1, LS1, SS2, LS2-LUC plasmid. (O-P) MCF-7 cells were co-transfected with miR-1254 and SS1, LS1, SS2 or LS2-LUC, and luciferase mRNA level (0) or luciferase activity (P) of FL/RL was determined. Dotted lines indicate the control level. Error bars indicate SEM. ${ }^{*} P<0.05$. Student's $t$-test except in F-I and $\mathbf{G}$, where $\chi$-square test is used. See also Supplementary information, Figure S3. 
A

miR-1254 3'- UGACGUCCGAGGUCGAAGGUCCGA -5

$\begin{array}{lll} & & \\ \text { miRancer-1254 } & 5^{\prime}- & \text { GGCUCCAGCUUCCA } \\ & 3^{\prime}- & \text { CCGAGGCGAGGU }\end{array}$

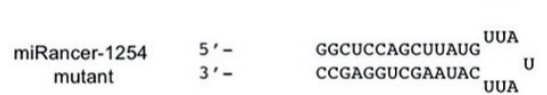

linear miR-1254 $\begin{gathered}\text { binding sequence } \\ \text { (miR-1254 sponge) }\end{gathered} 5^{\prime}-\quad$ GGCuccagcuuccagGcU $-3^{\prime}$

E

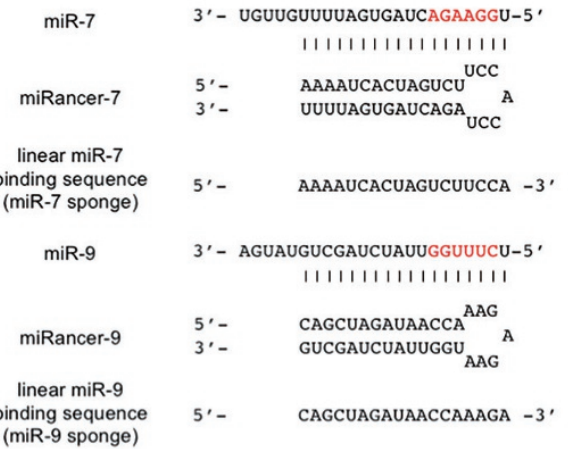

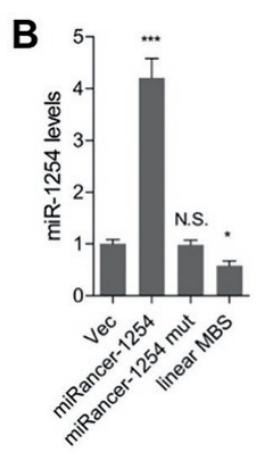

$\mathbf{F}$

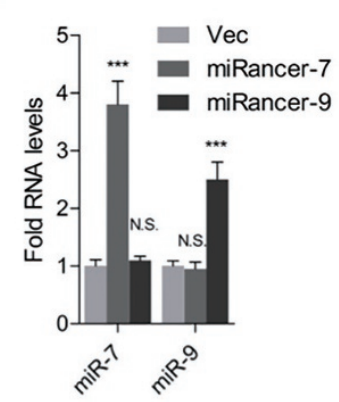

C ${ }^{2.0}{ }^{20} \quad$ D

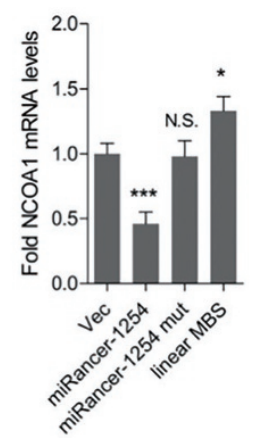

G

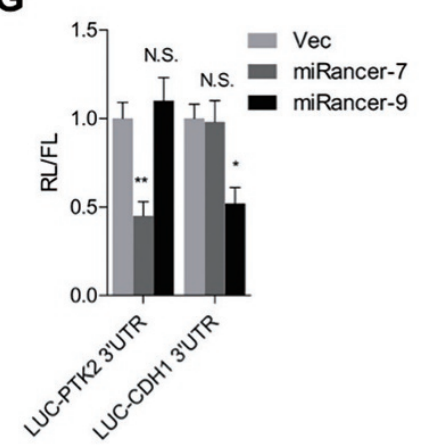

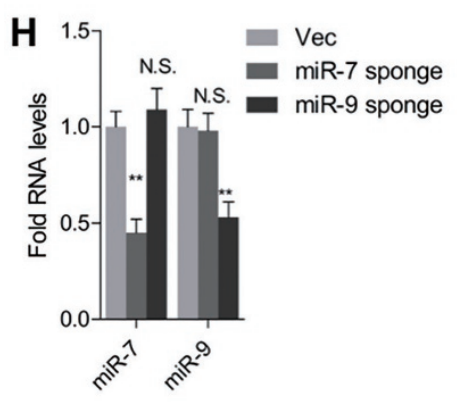
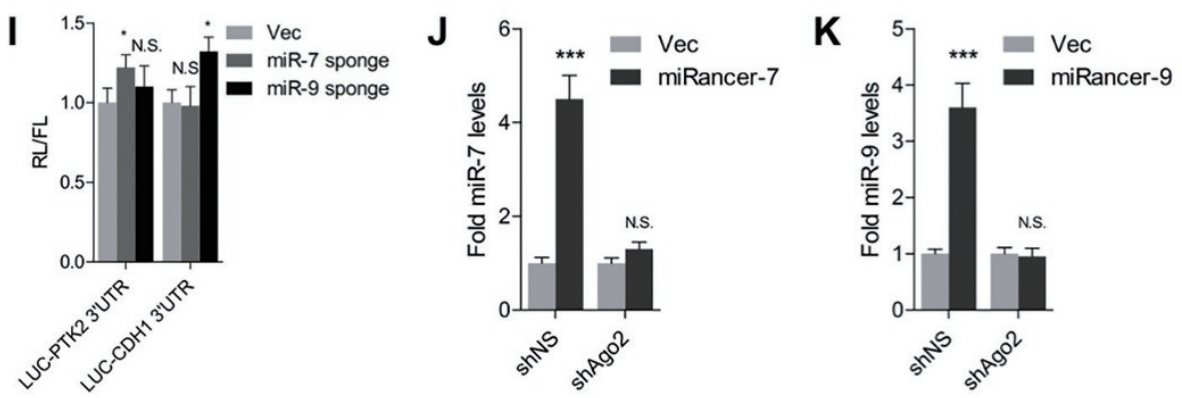

L

miRancer-7-LUC/ miR-7 sponge-LUC/ miRancer-9-LUC/ miR-9 sponge-LUC

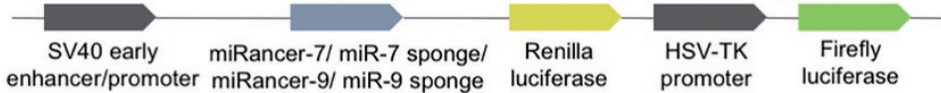

M

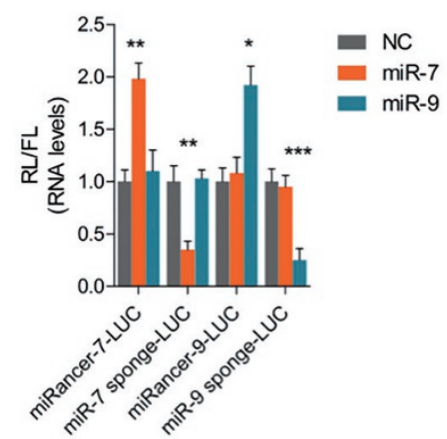

$\mathbf{N}$

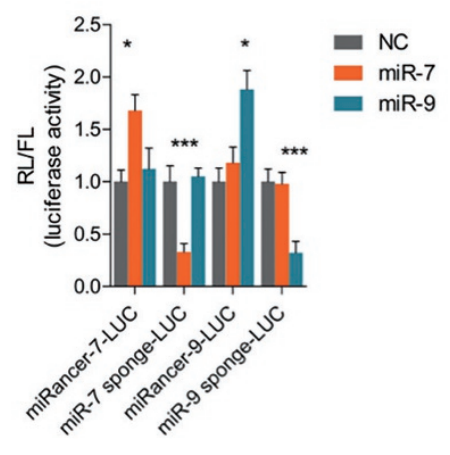


and activity (Figure 4I) of miR-7 or miR-9, respectively. We have also found that Ago2 was required for miRancer-potentiated miRNA expression (Figure 4J and 4K).

To determine whether the artificially designed miRancer could serve as a functional RNA motif responding specifically to the signal from the interacting miRNA, we constructed miRancer luciferase reporter plasmids by inserting the sequence of miRancer-7 or miRancer-9 in front of the luciferase gene (Figure 4L). We demonstrated that miR-7 or miR-9 specifically increased the reporter activity and luciferase mRNA level of their respective miRancer-containing reporter plasmid, whereas the replacement with cognate linear miRNA-binding sequence in the reporter plasmids resulted in reduced reporter activity and luciferase mRNA level (Figure $4 \mathrm{M}$ and $4 \mathrm{~N}$ ). Thus, the miRancer sequence embedded in the $5^{\prime}$ UTR as a functional RNA motif could be readily used as the specific sensor of its cognate miRNA for enhancing the expression of its host RNA.

\section{CCAR1 5' UTR overrides tamoxifen resistance}

To ascertain whether C5U encompassing two miRancer-like motifs could act as an independent regulator via its interaction with miR-1254, we first sought to determine the roles of miR-1254 in $\mathrm{ER}^{+}$breast cancer cells. Three lines of evidence suggested that miR-1254 is a tumor suppressor. First, miR-1254 suppressed cell growth and promoted apoptosis (Supplementary information, Figure S4). Second, miR-1254 suppressed epithelial-mesenchymal transition (EMT) and stem celllike characteristics in breast cancer cells (Supplementary information, Figure S5A-S5H). Third, xenograft studies showed that miR-1254 antagonism conferred breast cancer cells with enhanced tumor-initiating capacity in vivo as well as increased local invasion in primary tumors and pulmonary metastases (Supplementary information, Figure S5I-S5N).
EMT and stem-cell like traits have been implicated in the anti-estrogen sensitivity of $\mathrm{ER}^{+}$breast cancer cells [25-27]. Consistently, miR-1254 antagonism in $\mathrm{ER}^{+}$ breast cancer cells resulted in a reduced dependence on estrogen for growth and significant resistance to tamoxifen in vitro (Figure $5 \mathrm{~A}-5 \mathrm{~F}$ and Supplementary information, Figure S6A-S6F) and in vivo (Figure 5G-5I). Sections from tumors generated by cells carrying miR1254 sponge exhibited a higher percentage of Ki67-positive cells and a lower percentage of TUNEL-positive cells compared with control tumors, in the presence or absence of tamoxifen treatment (Supplementary information, Figure S6G-S6J). Interestingly, a substantial reduction of miR-1254 levels was also observed in two established prolonged endocrine therapy-mediated tamoxifen resistant (TAM-R) cell models [27, 28] (Supplementary information, Figure S6K and S6L), suggesting that miR1254 depletion might contribute to acquired tamoxifen resistance in $\mathrm{ER}^{+}$breast cancer cells. Predictably, miR1254 restoration in TAM-R cells restored sensitivity to tamoxifen (Figure $5 \mathrm{~J}$ to $5 \mathrm{~L}$ and Supplementary information, Figure S6M to S6O). miR-1254 restoration in TAM-R cells also substantially reduced their migratory capacity and stem cell-like population (Figure 5M5P and Supplementary information, Figure S6P-S6S). Collectively, these observations indicate that miR-1254 potently affects the oncogenicity, metastasis, stem-like characteristics and tamoxifen resistance of $\mathrm{ER}^{+}$mammary carcinoma cells in vitro and in vivo.

Consistent with its capacity to enhance miR-1254 expression, forced expression of C5U using CEG plasmid in TAM-R cells potently restored cells' sensitivity to tamoxifen as demonstrated by both in vitro assays (Figure 5Q and Supplementary information, Figure S6T) and xenograft studies (Figure 5S-5V and Supplementary information, Figure S6V-S6X). These data suggest CCAR1 $5^{\prime}$ UTR can serve as an independent functional unit to

Figure 4 Rational design and characterization of miRancer. (A) Alignment between miR-1254, miRancer-1254, seed region match sequence mutant of miRancer-1254 (miRancer-1254 mut) and linear miR-1254-binding sequence (linear MBS). (B-D) miR-1254 levels (B), RL/FL of miR-1254 reporter (C) and NCOA1 mRNA levels (D) upon transfection with pSilencer 4.1-CMV (Vec), miRancer-1254, miRancer-1254 mut or linear MBS. Error bars indicate SEM. ${ }^{\star} P<0.05$, ${ }^{* *} P<0.01$, ${ }^{* *} P<0.001$, N.S., not significant. (E) Alignment between miR-7, miRancer-7, miR-7 sponge, miR-9, miRancer-9 and miR-9 sponge. (F) miR7 and miR-9 levels by qPCR after transfection with pSilencer 4.1-CMV (Vec), miRancer-7, miRancer-9 for 48h. Error bars indicate SEM. ${ }^{* *} P<0.001$. (G) RL/FL of LUC-PTK2 3' UTR or LUC-CDH1 3' UTR after co-transfection with Vec, miRancer-7 or miRancer-9. Error bars indicate SEM. ${ }^{*} P<0.05,{ }^{* *} P<0.01$. (H) miR-7 and miR-9 levels by qPCR after transfection with Vec, miR-7 sponge or miR-9 sponge. Error bars indicate SEM. ${ }^{* *} P<0.01$. (I) RL/FL of LUC-PTK2 3' UTR or LUC-CDH1 3' UTR after co-transfection with Vec, miR-7 sponge or miR-9 sponge. Error bars indicate SEM. ${ }^{*} P<0.05$. (J-K) Ago2 depletion abrogates miRancer-7- or miRancer-9-mediated upregulation of miR-7 or miR-9. Error bars indicate SEM. ${ }^{* * *} P<0.001$. (L) Schematic diagram of the miRancer-7-LUC, miR-7 sponge-LUC, miRancer-9-LUC, miR-9 sponge-LUC plasmid. (M-N) mRNA levels (M) or luciferase activity (N) of FL/RL of miRancer-7-LUC, miR-7 sponge-LUC, miRancer-9-LUC, miR-9 sponge-LUC upon miR-7 or miR-9 overexpression. Error bars indicate SEM. ${ }^{*} P<0.05$, ${ }^{* *} P<0.01,{ }^{* * *} P<0.001$. Student's $t$-test. 

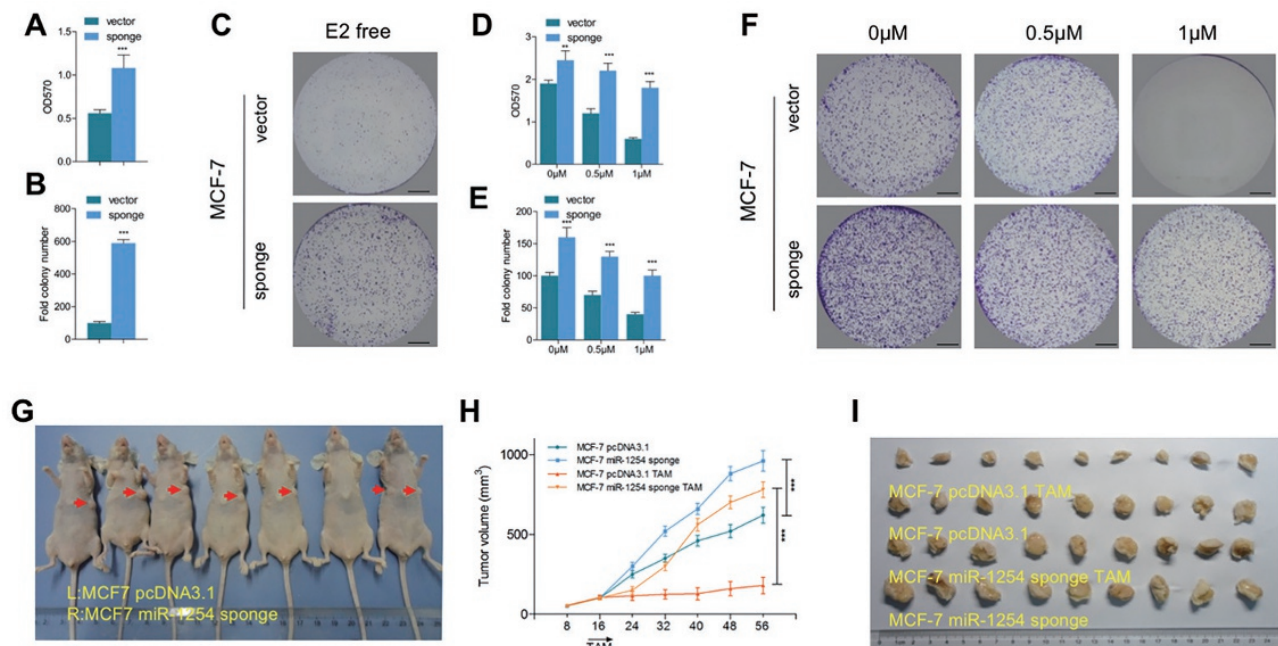

H

I
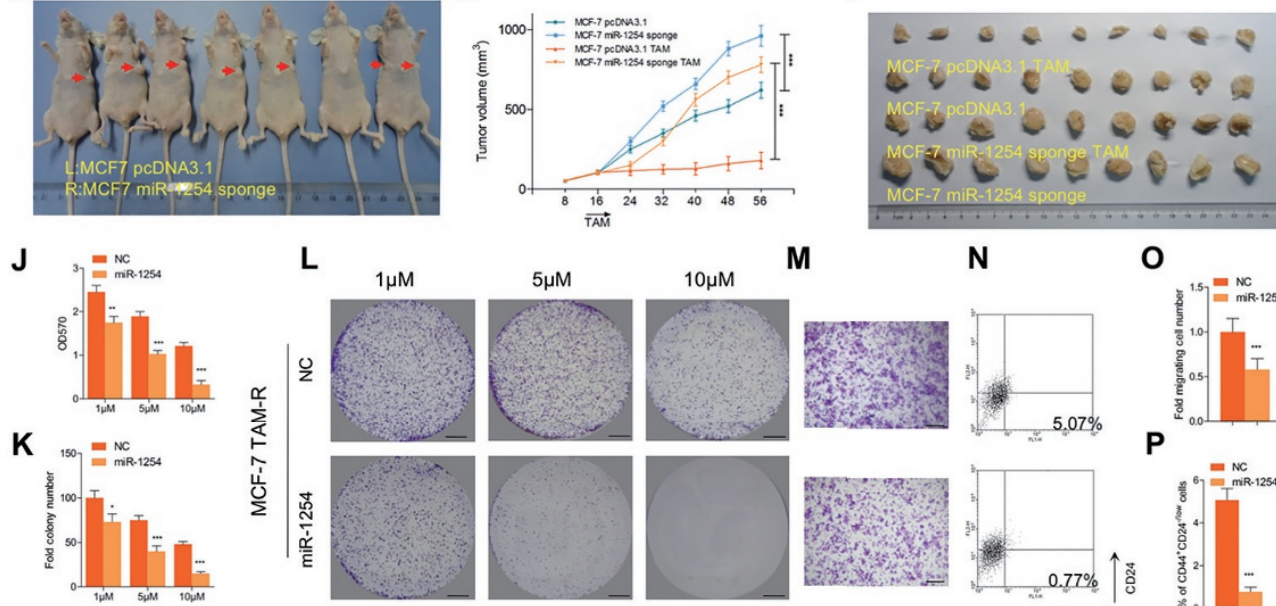

M N
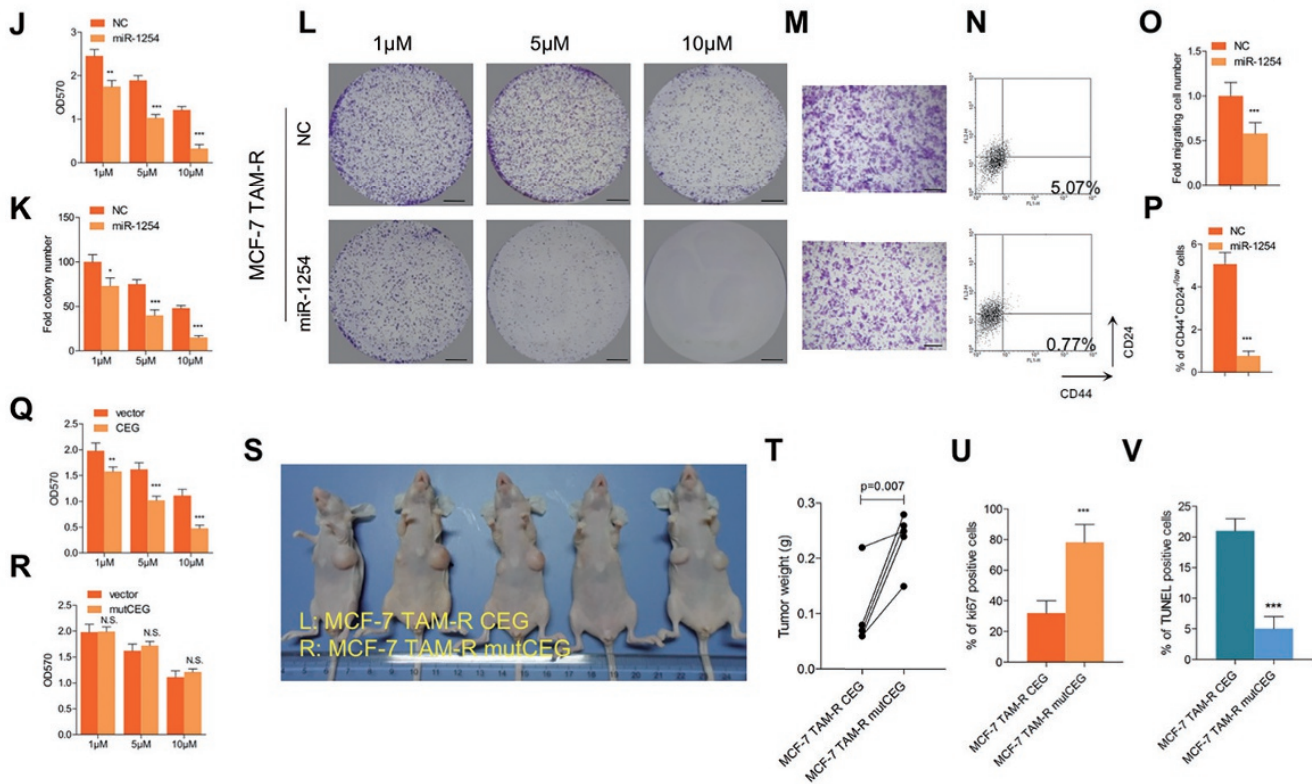

Figure 5 Functional characterization of miR-1254 and CCAR1 5' UTR in breast cancer cells. (A-C) MCF-7 cells were transfected with vector or miR-1254 sponge and deprived of estrogen for 8 days. Cell viability was measured by MTT assay (A), foci formation in soft agar (B) and monolayer culture (C). Scale bars represent $5 \mathrm{~mm}$. Error bars indicate SEM. ${ }^{* * *} P<0.001$. (D-F) MCF-7 cells were transfected with vector or miR-1254 sponge and treated with different doses of tamoxifen. Cell viability was measured by MTT assay (D), foci formation on soft agar (E) and monolayer culture (F). Scale bars represent 5 $\mathrm{mm}$. Error bars indicate SEM. ${ }^{* * *} P<0.001$. (G) Tumors (red arrows) form after orthotopic injection of MCF-7 pcDNA3.1 cells or MCF-7 miR-1254 sponge cells in nude mice without estrogen supplement for 40 days. (H-I) Growth curves $(\mathbf{H})$ and final sizes (I) of tumors derived from MCF-7 pcDNA3.1 cells or MCF-7 miR-1254 sponge cells orthotopically injected in nude mice with tamoxifen treatment for 40 days after the average tumor volume has reached $100 \mathrm{~mm}^{3}$ with estrogen supplementation. (J-L) MCF-7 TAM-R cells were transfected with NC or miR-1254 and further treated with different doses of tamoxifen for another 8 days. Cell viability was measured by MTT assay (J), foci formation on soft agar (K) and monolayer culture (L). Scale bars represent $5 \mathrm{~mm}$. Error bars indicate SEM. ${ }^{*} P<0.05,{ }^{* *} P<0.01$, ${ }^{* *} P<0.001$. (M-N) Transwell assay (M) measuring cell migration and FACS analysis (N) measuring CD44 and CD24 expression in MCF-7 TAM-R cells transfected with NC or miR-1254. Scale bars represent $100 \mu \mathrm{m}$. (O-P) Quantification of relative migrating cell number (O) and average of CD44 ${ }^{+} /$ CD24 ${ }^{- \text {llow }}$ population $(\mathbf{P})$ in three independent experiments. Error bars indicate SEM. ${ }^{* * \star} P<0.001$. (Q-R) MTT assay measuring viability of MCF-7 TAM-R cells transfected with vector or CEG $(\mathbf{Q})$, or vector or mutCEG (R) and treated with different doses of tamoxifen for 8 days. Error bars indicate SEM. ${ }^{* *} P<0.01,{ }^{* * *} P<0.001$. (S) Tumors derived from MCF-7 TAM-R CEG or MCF-7 TAM-R mutCEG in nude mice. (T-V) Tumor weight (T), cell proliferation measured by Ki67 staining (U), and cell apoptosis measured by TUNEL staining (V) of tumors derived from MCF-7 TAM-R CEG or MCF-7 TAM-R mutCEG cells. Error bars indicate SEM. $P=0.007$ in $\mathbf{T} ;{ }^{* * *} P<0.001$ in $\mathbf{U}$ and $\mathbf{V}$. Student's $t$-test except in $\mathbf{T}$, where Wilcoxon test is used. See also Supplementary information, Figure S4-S6. 
regulate tamoxifen sensitivity in $\mathrm{ER}^{+}$breast cancer cells. Transfection of mutCEG with mutant miR-1254-binding sites did not confer sensitivity to tamoxifen (Figure 5R and Supplementary information, Figure S6U), indicating C5U's activity is mainly mediated by its interaction with miR-1254. Together our results demonstrate that CCAR1 5' UTR which encompasses two miRancer-like motifs can override tamoxifen resistance in $\mathrm{ER}^{+}$breast cancer cells by serving as a natural miRancer of miR-1254.

\section{Trans-regulation between 5' UTR and 3' UTRS}

To elucidate the mechanism by which miR-1254 and C5U modulate anti-estrogen sensitivity, we utilized miRanda [29] and TargetScan [30] to predict potential miRNA target genes. Gene ontology analysis [31, 32] indicated the involvement of NCOA1, NCOA3, EGFR, $E R B B 2$ and $S N A I 1$ as candidate genes, all of which are well-established positive regulators of anti-estrogen resistance [33-35] and/or EMT [27, 36, 37] (Figure 6A). The full-length fragments of their 3' UTRs were cloned into luciferase reporter plasmids and co-transfected with miR-1254 mimics. We observed that miR-1254 markedly repressed the relative luciferase activities from the $3^{\prime}$ UTR of these genes, whereas mutation of the partially complementary miR-1254 sites in these $3^{\prime}$ UTRs abrogated the responsiveness to miR-1254 (Figure 6B). Furthermore, miR-1254 overexpression or antagonism significantly reduced or increased expression of these target genes, respectively (Figure 6C and Supplementary information, Figure S7B). miR-1254-mediated repression of these targets was also verified in vivo by immunohistochemistry (IHC) staining of formalin-fixed paraffin-embedded tumor sections derived from the xenograft studies (Supplementary information, Figure S7D). Thus, all of these genes are bona fide targets of miR-1254.

Consistent with the reduced miR-1254 level in TAM-R cells compared with the parental cells, the expression of miR-1254 target genes, all of which were well-established positive regulators of anti-estrogen resistance, was elevated (Supplementary information, Figure S7A). In contrast, miR-1254 restoration in TAM-R cells significantly reduced their expression. As the natural miRancer of miR-1254, forced expression of CCAR $15^{\prime}$ UTR by CEG significantly reduced the luciferase activity of reporters containing the $3^{\prime}$ UTR of miR-1254 target genes and the target gene expression in TAM-R cells (Figure $6 \mathrm{D}, 6 \mathrm{E}$ and $6 \mathrm{H}$ and Supplementary information, Figure S7C). This effect was abrogated when CEG was replaced by mutCEG (Supplementary information, Figure S7F and $\mathrm{S} 7 \mathrm{G}$ ), suggesting CCAR1 5' UTR modulates the expression of miR-1254 target genes via its interaction with miR-1254. Conversely, CCAR1 depletion by pooled
shRNAs in $\mathrm{ER}^{+}$breast cancer cells increased both 3' UTR reporter activity and expression of the miR-1254 target genes (Figure 6F-6H), leading to a loss of cellular sensitivity to tamoxifen (Supplementary information, Figure $\mathrm{S} 7 \mathrm{H})$. We also quantified the absolute copy numbers of miR-1254 and its downstream targets in tamoxifen-resistant cells and their parental cells, and found the levels of CCAR1 5' UTR and miR-1254 transcripts were significantly higher in parental cells (Supplementary information, Figure S7E). A positive correlation between miR-1254 and CCAR1 as well as a negative correlation between miR-1254 and other targets were observed. Of note, depleting miR-1254 target genes in parental cells compared with tamoxifen-resistant cognates correlated with a higher expression of miR-1254 and CCAR1. Thus, CCAR1 5' UTR acts as a natural trans-acting regulator of miR-1254 targets in $\mathrm{ER}^{+}$breast cancer cells.

The reciprocal signaling elicited by the 3' UTRs of miR-1254 targets to modulate CCAR1 5' UTR was also determined by expression of plasmids containing the $3^{\prime}$ UTR of miR-1254 target genes. Strikingly, decreased expression of CCAR1 and miR-1254 compared with the vector-transfected cells was observed (Supplementary information, Figure S7I and S7J). Notably, only wildtype but not mutant 3' UTRs of target genes significantly decreased CCAR1 and miR-1254 levels, suggesting that these 3 UTRs interact with miR-1254 to cross-regulate the CCAR1 5' UTR. Consistent with this finding, depletion of target genes using siRNA significantly increased the expression of CCAR 1 mRNA and miR-1254 (Supplementary information, Figure S7K and S7L). These data suggest that CCAR1 and miR-1254 target genes can cross-regulate the expression of each other via their respective $5^{\prime}$ UTR or $3^{\prime}$ UTRs.

\section{Discussion}

We present a novel regulatory paradigm by which miR-1254 interacts with the 5' UTR of its host gene CCAR1 mRNA in association with Ago2/miRISC, stabilizing each other at the post-transcriptional level and resulting in elevated expression of both molecules. In contrast to the contemporary model, wherein miRNA binds to the linear or unstructured MBSs of the targets, we demonstrate herein targeting of two highly structured miR-1254-binding sites on CCAR1 by miR-1254. Interestingly, a consistent base-paring pattern was observed in the interaction between the two MBSs on CCAR1 and the seed region of miR-1254, the most distinctive feature of which might be the consecutive miR-1254-binding sequences spanning the stem and loop/bulge regions. It is fascinating to note that miR-1254 could also enhance 
A

TargetScan:3824 targets

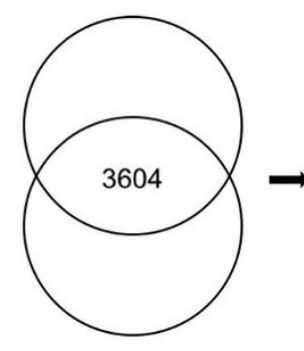

miRanda:9553 targets

B

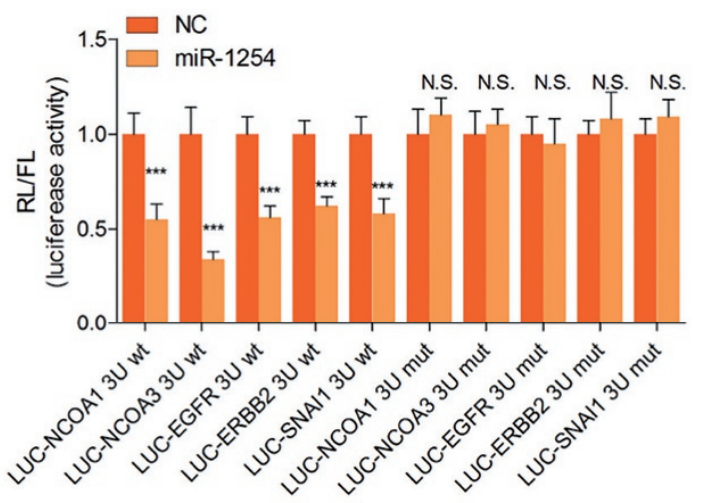

D
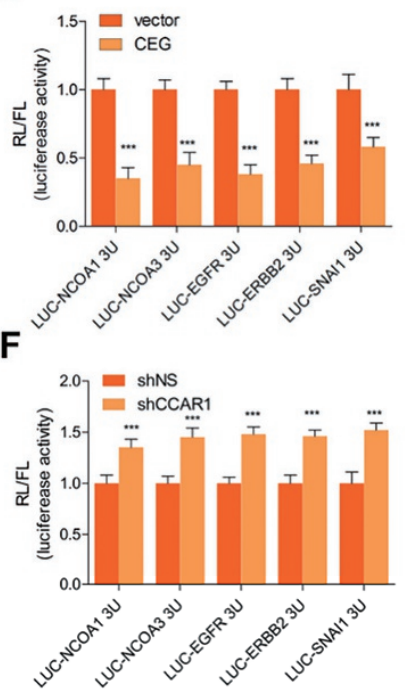

E

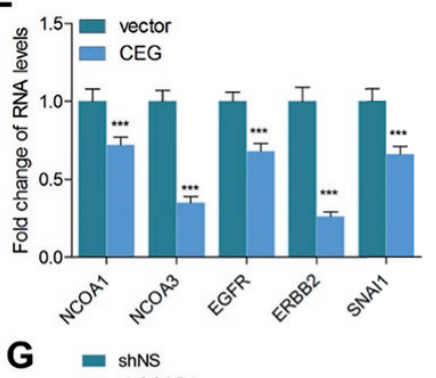

G $=$ snNS

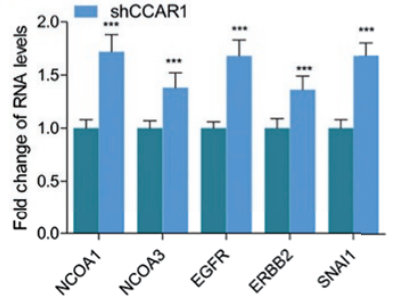

C

NCOA 1

NCOA3

EGFR

ERBB2

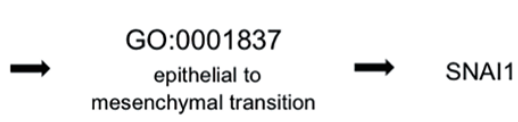

SNAI1

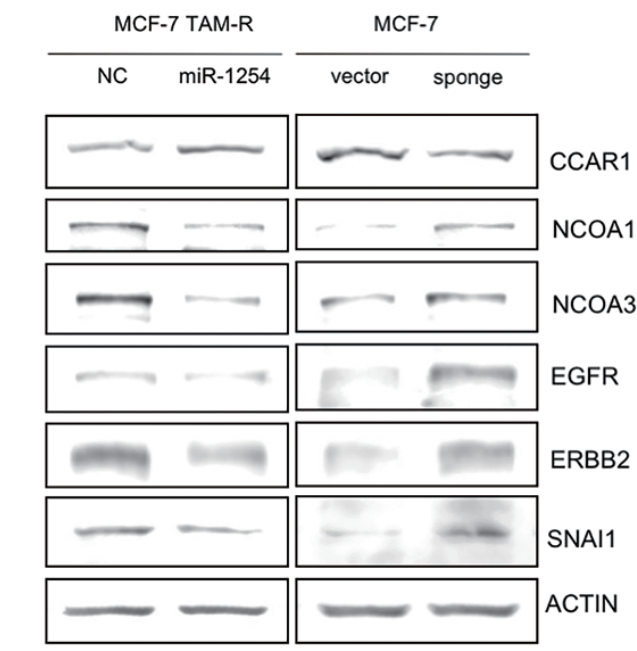

H

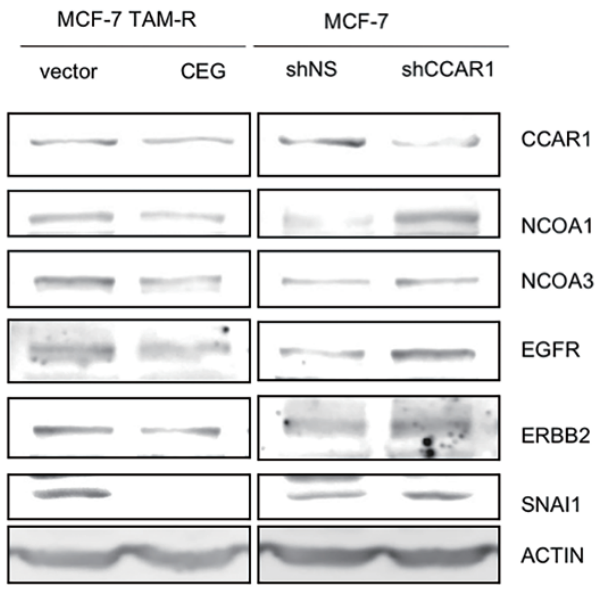

Figure 6 Mechanistic characterization of miR-1254 and CCAR1 5' UTR in modulating cellular sensitivity to tamoxifen. (A) miR-1254 targets predicted by TargetScan and miRanda with further GO analysis. NCOA1, NCOA3, EGFR, ERBB2 and SNA11 are selected. (B) Relative luciferase activity of wild-type or mutant 3' UTRs with forced expression of miR-1254. Error bars indicate SEM. ${ }^{* * *} P<0.001$, N.S., not significant. (C) Western blot analysis of CCAR1- and miR-1254-targeted genes upon forced expression of miR-1254 in MCF-7 TAM-R cells or miR-1254 antagonism in MCF-7 cells for 48h. (D-E) Relative luciferase activity of $3^{\prime}$ UTR luciferase reporter (D), mRNA levels (E) of miR-1254-targeted genes in MCF-7 TAM-R cells transfected with CEG or vector. Error bars indicate SEM. ${ }^{* *} P<0.001$. (F-G) Relative luciferase activity of $3^{\prime}$ UTR luciferase reporter (F) and mRNA levels (G) of miR-1254-targeted genes in MCF-7 TAM-R cells transfected with shNS or shCCAR1. Error bars indicate SEM. ${ }^{* *} P<0.001$. (H) Protein levels of indicated genes upon forced expression of CEG in MCF-7 TAM-R cells or depletion of CCAR1 in MCF-7 cells. Student's t-test. See also Supplementary information, Figure S7. 
TP53INP1 expression via a similar base-pairing pattern, wherein the miR-1254-binding sites on TP53INP1 exhibits a similar higher order structure. This particular hairpin structure was further verified to be critical for miR-1254enhanced expression of CCAR1 and TP53INP1 as well as other miRNAs (miR-7 and miR-9) that promoted the expression of cognate miRancer-containing transcripts. Thus, MBSs on 5' UTR of target mRNA with defined structural and sequence features could direct the interacting miRNA as an enhancer instead of a repressor. It should be noted that the paradigm presented herein is intrinsically differently from other miRNA-mediated mechanisms to elevate gene expression, which employ either transcriptional or translational activation $[8,9]$, wherein miRNA binds directly to the $5^{\prime}$ UTR of mRNA, enhancing the association of the RNA helicase DHX9 to the mRNA transcript to promote transcription [8], or binds immediately downstream of the regulatory 5'TOP motif to alleviate translational repression [9].

It is fascinating to observe that the extracted miR-1254-targeting sequence could serve as both a functional RNA motif and an independent RNA functional unit. On one hand, it behaves as a RNA sensor for miR1254 by transmitting its signal to enhance CCAR 1 or TP53INP1 expression; on the other hand, it serves as a functional RNA fragment by interacting with miR1254 to enhance its expression. Strikingly, the linear miR-1254-targeting RNA sequences as either motif or functional RNA unit exhibited completely opposite roles to their prototypes, further verifying the pivotal role of the RNA structure of miRNA-targeting sequence in determining the outcomes of target RNA binding by a miRNA. The extension of the miR-1254/CCAR1 paradigm to miR-1254/TP53INP1 and particularly to other miRNAs and their respective structured targeting transcripts (Supplementary information, Figure S3) suggests this mechanism could be commonly used in RNA biology. Thus we have discovered additional complexity to the canonical model of miRNA: mRNA interaction, wherein miRNAs bind to the unstructured MBSs of target RNAs, leading to mutually repressed expression or activity. As a further refined model of miRNA function, we propose herein that miRNAs also bind to target RNAs with highly structured MBSs, leading to reciprocal stabilization (Figure 7A).

By further extraction and extrapolation of the features of miR-1254-targeting sequences as independent functional RNA units, an artificial miRNA enhancer termed "miRancer" was generated, which can specifically increase the expression and activity of the endogenous miRNAs of interest. Fascinatingly in reciprocity, miRancer motif embedded within the 5' UTR of the coding RNA transcript could readily mediate its cognate miRNA-boosted gene expression. The exact mechanism by which miRancer sequence embedded in the 5' UTR enhances the expression of its host RNA would need further investigation. However, it is interesting to note that miR-122 slows the decay of HCV RNA through a process involving the recruitment of Ago2 to its 5' UTR [7] and the $5^{\prime}$ exonuclease Xrn1 [38], leading to a stabilized viral genome and increased production of infectious virions.

RNA structure appears to be critical in determining the "enhancer" function of miRancer and miRancer motif. As Argonaute protein is a limiting factor for the stability of miRNA $[39,40]$ as well as target RNA [1, 3, 7] (Figure 3A-3I), effort is needed to further define the structural and mechanistic basis of miRNA:miRancer reciprocal stabilization in the context of Ago2/miRISC. It is intriguing to note that a local triple-helix RNA structure or triplex RNA structure, wherein both Watson-Crick pairing and/or Hoogsteen pairing have been known to be utilized, is critical for the enhanced RNA stability [41, 42].The possible formation and functional implication of a localized triple-helix RNA structure by miR-1254 and its structured binding sites warrant further investigation.

Unlike its conventional role as a cis-regulatory element, the unexpected discovery of CCAR $15^{\prime}$ UTR as an independent cellular regulator might represent for the first such report that $5^{\prime}$ UTR can serve as an independent functional unit, although a number of studies have suggested that $3^{\prime}$ UTRs independently regulate cellular events $[43,44]$. For example, recently discovered competitive endogenous RNAs (ceRNAs) are characterized by the symmetrical trans-acting regulation among $3^{\prime}$ UTRs of different coding genes to modulate various cellular behaviors $[45,46]$. In our studies, the bi-directional regulation between the 5' UTR of CCAR1 and 3' UTRs of miR-1254-target genes represents a novel regulatory paradigm which allows asymmetrical trans-regulation among different genes (Figure 7B).

It is of further interest to observe that the CCAR1 5' UTR containing two miRancer motifs acts like an endogenous or natural miRancer of miR-1254 to resensitize tamoxifen-resistant $\mathrm{ER}^{+}$breast cancer cells by increasing miR-1254 expression and subsequently suppressing 5 pro-oncogenic targets via their $3^{\prime}$ UTRs. It is plausible that the natural miRancer might have evolved to sequence-specifically enhance miRNAs to modulate their associated cellular behaviors. This carries implications for the development of novel therapeutic approaches by using either miRancer-motif containing RNA fragments or miRancer molecules. 
A

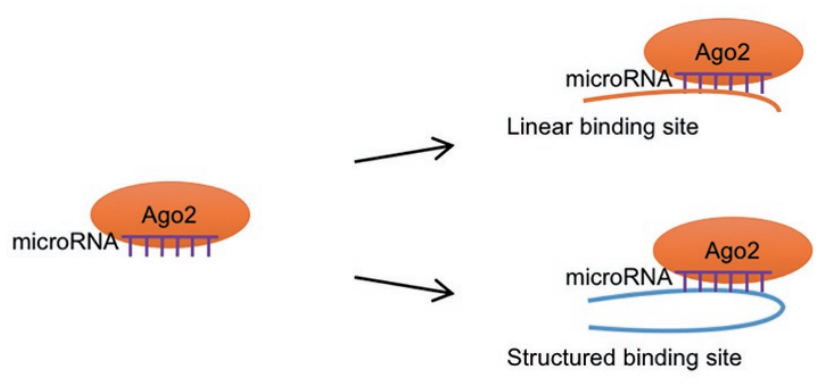

ताTाTा

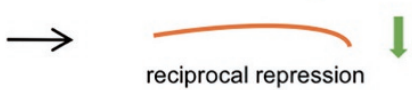

reciprocal repression

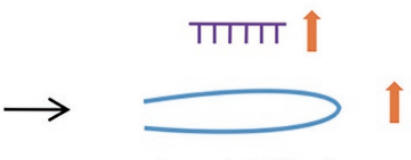

reciprocal stabilization

B

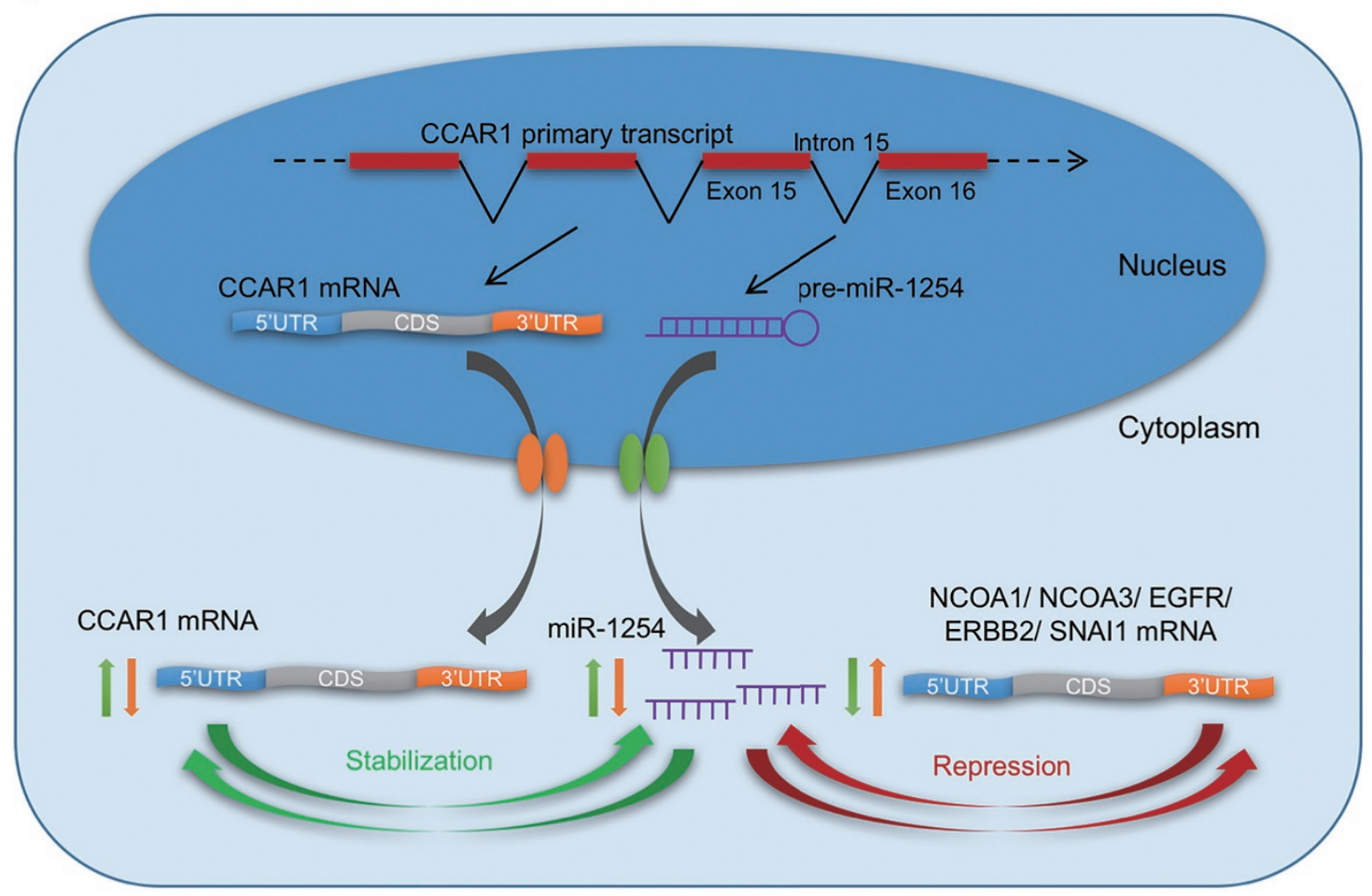

Figure 7 Diagram of working model. (A) Ago2 facilitates miRNA binding to a linear or structured miRNA-binding site to promote reciprocal functional repression (green arrows) or stabilization (orange arrows), respectively. (B) Molecular crosstalk among CCAR1 5' UTR, miR-1254 and miR-1254-targeted genes. CCAR1 mRNA increases miR-1254 level, which causes an enhanced repression of miR-1254 target gene expression (orange arrows), while miR-1254 target gene mRNA reduces miR1254 level, which leads to a repression of CCAR1 mRNA (green arrows).

\section{Materials and Methods}

\section{Cell lines}

MCF-7 and T-47D cells were purchased from ATCC and the authentication of cell lines by STR profile analysis was performed by a third party (Genewiz; Supplementary information, Data S1), MCF-7 TAM-R and T-47D TAM-R cells were generated as previously described [47]. All cell lines were cultured in RPMI-1640 medium with $10 \%$ FBS (Gibco) and $1 \%$ Pen/Strep (Invitrogen) at $37^{\circ} \mathrm{C}$ in a $5 \% \mathrm{CO}_{2}-95 \%$ air incubator.

\section{Generation of stable cell lines}

Expression vectors were transfected into cells in $6 \mathrm{~cm}$ flasks for
$24 \mathrm{~h}$. Cells were then re-plated into $10 \mathrm{~cm}$ plates in the presence of $800 \mu \mathrm{g} / \mathrm{ml} \mathrm{G} 418$ for selection over 2 weeks.

\section{Patients and specimens}

The patient population consisted of 57 consecutive patients with invasive mammary ductal carcinoma who underwent surgery at the First Affiliated Hospital of Anhui Medical University (Hefei, Anhui, People's Republic of China) between 2001 and 2002. All patients were Han Chinese female. 34 carcinoma tissues and 23 normal mammary tissue next to tumor were obtained. The pathohistological diagnoses of the specimens were consistent with breast neoplasm in accordance with WHO guidelines [48]. Histology grade was based on the Scarff-Bloom-Richardson system 
[49]. The study procedures were in accordance with the ethical standards of the responsible committee on human experimentation and with the Helsinki Declaration of 1975, revised in 2008.

\section{Reagents}

Protein electrophoresis reagents were from Bio-Rad Laboratories. Tamoxifen and actinomycin D were from Sigma.

\section{Animal Studies}

All studies were conducted in accordance with the Guide for the Care and Use of Laboratory Animals, published by the NIH (NIH Publication 85-23, revised 1985). An Institutional Animal Care and Use Committee (IACUC) approved all animal protocols.

For orthotopic injection, $5 \times 10^{2}-5 \times 10^{5}$ cells (for limiting dilution analysis) or $5 \times 10^{6}$ cells (for others) were unilaterally injected into the second mammary fat pad of 5- to 6-week-old female $\mathrm{BALB} / \mathrm{c}$ nude mice with subcutaneously implanted long-release $17-\beta$ estradiol pellets (Innovative Research of America). Tamoxifen treatment was applied by implanting subcutaneously a long-release tamoxifen pellet (Innovative Research of America) when the tumors reached a volume of $100 \mathrm{~mm}^{3}$. Tumor growth rates were analyzed by measuring tumor length (L) and width (W), and calculating tumor volume based on the formula: volume $\left(\mathrm{V}, \mathrm{mm}^{3}\right)=\mathrm{L} \times$ $\mathrm{W}^{2} \times 1 / 2$. Tumors were surgically resected 8 weeks after injection and tumor weight was measured using a scale.

For experimental metastasis assays, 5- to 6-week-old female $\mathrm{BALB} / \mathrm{c}$ nude mice were injected with $2 \times 10^{6}$ cells (resuspended in $100 \mu \mathrm{l}$ PBS) via the lateral tail vein. 8 weeks later, animals were sacrificed and lungs were harvested. Some tissues were fixed overnight in 4\% paraformaldehyde ( $\mathrm{pH} 7.4$ ), embedded in paraffin and cut into $5 \mu \mathrm{m}$-thick sections for histological studies; other tissues were frozen at $80{ }^{\circ} \mathrm{C}$ in RNALater (Ambion) for RNA extraction and real-time PCR analysis for hHPRT/mGAPDH to evaluate metastasis.

\section{shRNA expression}

shRNAs against CCAR1, Ago1, Ago2, NCOA1, NCOA3, EGFR, ERBB2, SNAI1, Drosha, Dicer were cloned in pSilencer 4.1-CMV puro (Ambion) following manufacturer's instruction; target sequences are listed in Supplementary information, Table S1.

\section{Flow cytometry}

Annexin V staining was performed according to manufacturer's instruction (Bio vision). To study the cell cycle, propidium iodide staining was used as previously described [50]. CD44/CD24 staining was performed as previously described [51].

\section{Cell function assay}

MTT assay, soft agar assay, colony formation assay and 2-D matrigel culture were performed as described previously [52-54]. Transwell migration assay was performed in $8-\mu \mathrm{m}$ pore chambers (Corning Costar) as described previously [55]. Values for cell migration are expressed as the average number of cells per microscopic field. For mammosphere culture, cells were harvested in $0.05 \%$ trypsin-EDTA and carefully resuspended in DMEM/F12 (Invitrogen) supplemented with B27 (1:50, Gibco), bovine serum albumin (0.4\%, Sigma), EGF (20 ng/ml, Upstate), bFGF (20 $\mathrm{ng} / \mathrm{ml}$, Peprotech), insulin $(5 \mu \mathrm{g} / \mathrm{ml}$, Sigma $)$, penicillin-strepto- mycin (Gibco), L-glutamine (Gibco). To induce sphere formation, 5000 cells were plated into poly (2-hydroxyethyl metacrylate)(Polyhema, Sigma) coated six-well plates to prevent the cells from attaching to the surface. For self-renewal assay, the spheres were dissociated into single cells after 7 days and seeded again as described previously [56]. For quantification, 1000 cells per well of the dissociated single cells were seeded in medium containing $1 \%$ methylcellulose (Sigma) in Polyhema-coated 96-well plates. Colonies $>75 \mu \mathrm{m}$ in diameter were counted after 7 days.

\section{Affinity purification for miR-1254-associated $m R N A$}

MCF-7 cells transfected with 3' biotin-tagged miR-1254 or controls were subject to affinity purification as previously described [57].

\section{Affinity purification for C5U associated miRNA}

Some modification of ChIRP [58] has been applied to determine the C5U-associated miRNAs. 3' biotin-tagged DNA probes against $\mathrm{C} 5 \mathrm{U}$ were synthesized: $\mathrm{C} 5 \mathrm{U}$ probe A, 5'-CGGCGCTTACTGGCTGACGTTCT-3'; C5U probe B, 5'-CCAACTTCCCCCTCTTCCTCCAC-3', and C5U probe C, 5'-TTCTATAGCATCGATCTGAGTCG-3'. 3' biotin-tagged probe against lacZ was synthesized: 5'-CAAACGACTGTCCTGGCCGTAAC-3'. All probes were compared with the human transcripts using the BLAST tool, and no more than 15-mer non-specific overlap was noticed. MCF-7 cells were grown to $\log$-phase in $10 \mathrm{~cm}$ plate and transfected with vector, CEG or mutCEG for $24 \mathrm{~h}$, rinsed once with room temperature $\mathrm{PBS}$, and fixed in $2 \mathrm{ml} 1 \%$ formaldehyde in PBS for $10 \mathrm{~min}$ at room temperature. Crosslinking was then quenched with $0.125 \mathrm{M}$ glycin for $5 \mathrm{~min}$. Cells were rinsed again with PBS, harvested into Falcon tubes, and pelleted at $800 \times \mathrm{g}$ for $3 \mathrm{~min}$. Cell pellets were resuspeded in $1 \mathrm{ml}$ swelling buffer $(0.1 \mathrm{M}$ Tris $\mathrm{pH}$ 7.0, $10 \mathrm{mM}$ KOAc, $15 \mathrm{mM} \mathrm{MgOAc})$, and 1\% NP-40, 1 $\mathrm{mM}$ DTT, $1 \mathrm{mM}$ PMSF, complete protease inhibitor mix (GE), and $0.1 \mathrm{unit} / \mathrm{ml}$ Superase-in (Ambion) were added for $10 \mathrm{~min}$ on ice. Cell suspension was then homogenized using a Dounce homogenizer and pelleted at $2500 \times \mathrm{g}$ for $5 \mathrm{~min}$. Suspension was diluted in two times the volume of hybridization buffer $(750 \mathrm{mM}$ $\mathrm{NaCl}, 1 \%$ SDS, $50 \mathrm{mM}$ Tris 7.0, $1 \mathrm{mM}$ EDTA, 15\% formamide with fresh DTT, PMSF, complete protease inhibitor mix, and Superase-in). $100 \mathrm{pmol}$ of the probe was added to $3 \mathrm{ml}$ hybridization buffer, which was then mixed by end-to-end rotation at room temperature for $4 \mathrm{~h}$. Streptavidin magnetic C1 beads (Invitrogen), before adding to the mixture containing the probe, were washed three times in wash buffer, blocked with $500 \mathrm{ng} / \mu \mathrm{l}$ yeast total RNA and $1 \mathrm{mg} / \mathrm{ml} \mathrm{BSA}$ for $1 \mathrm{~h}$ at room temperature, and washed three times again in wash buffer before resuspended in its original volume. $100 \mu \mathrm{l}$ washed/blocked $\mathrm{C} 1$ beads were added to $100 \mathrm{pmol}$ of the probe, and the entire reaction was mixed and incubated for another 30 min at $37^{\circ} \mathrm{C}$. Beads were captured by magnets (Invitrogen) and washed five times with $4 \mathrm{ml}$ wash buffer $(2 \times \mathrm{SSC}, 0.5 \% \mathrm{SDS}$, and fresh DTT and PMSF). After last wash, buffer was removed carefully using a P-10 pipette so that no trace volume was left behind. Beads were resuspended in 10 original volumes of RNA elution buffer (Tris 7.0, 1\% SDS) and boiled for $15 \mathrm{~min}$, followed by qRTPCR assay.

\section{$q R T-P C R$}

Total RNA was isolated (TRIZOL, Invitrogen), DNase-treated 
(DNase I, Fermentas), reverse-transcribed (Superscript III, Invitrogen) and analyzed by qPCR (SYBR Green, TAKARA) using specific primers (Supplementary information, Table S2). miRNA were isolated using mirVana miRNA isolation kit (Ambion), reverse transcribed with Superscript III (Invitrogen) using stem-loop RT primers and analyzed by qPCR (Taqman, TAKARA) using specific primers (Supplementary information, Table S2).

\section{Transcript copy number analysis}

To determine the transcript copy number, RNA was prepared from $5 \times 10^{6}$ cells using total RNA mini-prep kit (Axygen) following manufacturer's instruction. qRT-PCR was performed for CCAR1 5' UTR, NCOA1 3' UTR, NCOA3 3' UTR, EGFR 3' UTR, ERBB2 3' UTR, SNAI1 3' UTR and miR-1254 as described above. Threshold cycle $\left(\mathrm{C}_{\mathrm{T}}\right)$ values were compared with a 10 fold dilution series of linearized C5U-LUC, LUC-NCOA1 3' UTR, LUC-NCOA3 3' UTR, LUC-EGFR 3' UTR, LUC-ERBB2 3' UTR, LUC-SNAI1 3' UTR or a synthetic single-stranded miR1254 mimic (Genepharma). Moles of transcripts and copies of transcripts per cell were then calculated using standard stoichiometric methods. Confidence intervals were calculated using Student's $t$-test.

\section{Western blot}

Western blot analysis was performed using standard methods. Antibody details are described in Supplementary information, Table S3.

\section{Ago2-RNA coimmunoprecipitation}

MCF-7 CEG cells transfected with either negative control (NC) or miR-1254. MCF-7 cells were transfected with CEG or mutCEG. These cells were subject to Ago2-RNA immunoprecipitation as previously described [7].

\section{Luciferase reporter assays}

For CPE reporter assay, cells were seeded into 24-well plates in triplicate and transfected with CPE and pRL-TK (Promega), and further transfected with $\mathrm{NC}$, miR-1254 mimic, vector control, or miR-1254 sponge. $24 \mathrm{~h}$ later, luciferase activities were determined using the Dual-Luciferase Assay System following manufacturer's instruction (Promega). mRNA levels of firefly luciferase and Renilla luciferase were determined using qRT-PCR as above.

For luciferase reporter assay using psiCHECK2 reporter, cells were seeded into 24-well plates in triplicate and transfected with psiCHECK2 reporter and either NC, miR-1254 mimic, vector, or miR-1254 sponge. Luciferase activities and mRNA levels were determined as above.

\section{Transfection}

Cells were transfected with miRNA using Hiperfect (Qiagen) and with plasmid using Attractene (Qiagen) following manufacturer's instruction. Cells were subsequently subject to cell function assays as above or collected for RNA or protein as above.

\section{Histopathological analysis.}

After mice were euthanized by $\mathrm{CO}_{2}$ asphyxiation, lungs and tumors were collected and fixed overnight in $10 \%$ formalin. They were subsequently embedded, sectioned, and stained with haematoxylin and eosin (H\&E), Ki67, TUNEL, or other antibodies described in Supplementary information, Tables S3 and S4.

\section{Statistical testing}

Statistical significance was assessed by the Student's $t$-test, Wilcoxon test or $\chi$-square test as indicated by figure legends.

\section{Plasmid constructs and mutagenesis}

A synthetic DNA corresponding to CCAR1 5' UTR was inserted into psiCHECK2 (Promega) at Nhe I to create C5U-LUC. CCAR1 3' UTR was PCR amplified from MCF-7 cDNA and inserted into psiCHECK2 (LUC-C3U) between Xho I and Not I sites. C5U, EGFP CDS and GAPDH 3' UTR were PCR amplified from C5U-LUC, pIRES2-EGFP (Clontech) and MCF-7 cDNA, respectively; overlap PCR was then performed to generate CEG, which was subsequently inserted into pcDNA3.1 (+). 4 tandem repeats of bulged miR-1254 target sequence were synthesized and subcloned (HindIII + XhoI) into pcDNA3.1 as miR-1254 sponge [18], miR-1254 target sequence was synthesized and inserted (XhoI + NotI) into psiCHECK-2 as miR-1254 reporter. For expression of SS1, SS2, LS1, LS2, and miRancers, complementary oligonucleotides were annealed to form double-stranded DNA with sticky ends, which was ligated into pSilencer 4.1-CMV puro (Ambion) plasmid between BamHI and HindIII. For miRancer containing reporter and sponge-containing reporter, complementary oligonucleotides were annealed to form double-stranded DNA with sticky ends and ligated into psiCHECK2 at Nhe I site. For miRNA target reporters and 3' UTR expressing plasmid, the full-length 3' UTRs of NCOA1, NCOA3, EGFR, ERBB2, SNAI1 were PCR-amplified from MCF-7 cDNA and cloned into psiCHECK-2 at XhoI and NotI, and pcDNA3.1 (+) at BamHI and NotI, respectively. Site-directed mutagenesis was performed using the QuickChange II XL site-directed mutagenesis kit (Stratagene) to change the seed-matching sequences. All constructs were confirmed by DNA sequencing. The primers used are shown in Supplementary information, Table S5.

\section{Ago2 PAR-CLIP dataset analysis}

We downloaded a dataset of AGO2 PAR-CLIP raw sequencing files from NCBI Sequence Read Archive (SRA) database [59] (SRR1045082 [22]). Low-quality bases and adaptors were removed from raw sequences using Filter-seq.pl and Cutadapt, respectively. Clean reads were then collapsed to unique reads by Fastx toolkit. Unique reads were aligned to human reference genome (hg19) using Bowtie [60] with parameters -v 2 -m 10 -best -strata. We employed PARalyzer [61] to identify AGO2-binding sites, with read deep $\geq 5$ and unique locations of $\mathrm{T}$ to $\mathrm{C}$ conversions $\geq 1$. AGO2-binding sites overlapping with 5' UTR regions of human known coding genes were retrieved, and further scanned for potential miR-1254-binding sites, by seed matches including $1-7,2-8,1-6,2-7,3-8$ bases of the seed region, allowing for G-U wobble base pair. AGO2-binding sites overlapping with 5' UTRs and containing miR-1254-binding sites were verified by experiments.

\section{Acknowledgments}

We thank Dr Saraswati Sukumar for critical comments on the manuscript. We also thank Dr Zhaoyong Li, Ms Xinlei Gao and Mr Xiaolin Wang for technical assistance. This work was support- 
ed by the Ministry of Science and Technology (2012CB934002 and 2015CB943000) of China, the Chinese Academy of Sciences (XDA01040410 and KJZD-EW-L01-2), the National Natural Science Foundation of China (81272925, 81472494, 31471225, 31201022) of China, the Fundamental Research Funds for the Central Universities (WK2070000034), the Ministry of Education of China (20123402110007), and the Cancer Science Institute of Singapore, which received funds from the National Research Foundation and the Ministry of Education of Singapore.

\section{Author Contributions}

TZ and GL conceived the principle concept, TZ, GL, GS and XW designed the experiments. Constructs were cloned by GL, XW and WQ. XW and WQ performed qPCR and luciferase reporter assays. GL and XW performed cell function assays. GL and XS performed western blot and IHC. GL and XW performed animal studies. XW, XZ and XL performed cytometric assays. ZW, ST, $\mathrm{KD}, \mathrm{HS}, \mathrm{SG}$ and HY interpreted the data and provided advice. TZ, GL, PEL and GS wrote the manuscript.

\section{Competing Financial Interests}

The authors declare no conflict interests.

\section{References}

1 Di Leva G, Garofalo M, Croce CM. MicroRNAs in cancer Annu Rev Pathol 2014; 9:287-314.

2 Brummer A, Hausser J. MicroRNA binding sites in the coding region of mRNAs: extending the repertoire of post-transcriptional gene regulation. Bioessays 2014; 36:617-626.

3 Sharp PA. The centrality of RNA. Cell 2009; 136:577-580.

4 Pasquinelli AE. MicroRNAs and their targets: recognition, regulation and an emerging reciprocal relationship. Nat Rev Genet 2012; 13:271-282.

5 Bartel DP. MicroRNAs: target recognition and regulatory functions. Cell 2009; 136:215-233.

6 Pesole G, Mignone F, Gissi C, Grillo G, Licciulli F, Liuni S. Structural and functional features of eukaryotic mRNA untranslated regions. Gene 2001; 276:73-81.

7 Shimakami T, Yamane D, Jangra RK, et al. Stabilization of hepatitis $\mathrm{C}$ virus RNA by an Ago2-miR-122 complex. Proc Natl Acad Sci USA 2012; 109:941-946.

8 Liu M, Roth A, Yu M, et al. The IGF2 intronic miR-483 selectively enhances transcription from IGF2 fetal promoters and enhances tumorigenesis. Genes Dev 2013; 27:2543-2548.

9 Orom UA, Nielsen FC, Lund AH. MicroRNA-10a binds the $5^{\prime}$ UTR of ribosomal protein mRNAs and enhances their translation. Mol Cell 2008; 30:460-471.

10 Sengupta S, Jordan VC. Selective estrogen modulators as an anticancer tool: mechanisms of efficiency and resistance. $A d v$ Exp Med Biol 2008; 630:206-219.

11 Viedma-Rodriguez R, Baiza-Gutman L, Salamanca-Gomez $\mathrm{F}$, et al. Mechanisms associated with resistance to tamoxifen in estrogen receptor-positive breast cancer. Oncol Rep 2014; 32:3-15

12 Loo LW, Wang Y, Flynn EM, et al. Genome-wide copy number alterations in subtypes of invasive breast cancers in young white and African American women. Breast Cancer Res Treat 2011; 127:297-308.
13 Kozomara A, Griffiths-Jones S. miRBase: annotating high confidence microRNAs using deep sequencing data. Nucleic Acids Res 2014; 42:D68-73.

14 Kim JH, Yang CK, Heo K, Roeder RG, An W, Stallcup MR. CCAR1, a key regulator of mediator complex recruitment to nuclear receptor transcription complexes. Mol Cell 2008; 31:510-519.

15 Rishi AK, Zhang L, Yu Y, et al. Cell cycle- and apoptosis-regulatory protein-1 is involved in apoptosis signaling by epidermal growth factor receptor. J Biol Chem 2006; 281:13188-13198.

16 Down TA, Hubbard TJ. Computational detection and location of transcription start sites in mammalian genomic DNA. Genome Res 2002; 12:458-461.

17 Chien $\mathrm{CH}$, Sun YM, Chang WC, et al. Identifying transcriptional start sites of human microRNAs based on high-throughput sequencing data. Nucleic Acids Res 2011; 39:9345-9356.

18 Ebert MS, Neilson JR, Sharp PA. MicroRNA sponges: competitive inhibitors of small RNAs in mammalian cells. Nat Methods 2007; 4:721-726.

$19 \mathrm{Wu}$ X, Brewer G. The regulation of mRNA stability in mammalian cells: 2.0. Gene 2012; 500:10-21.

20 Kruger J, Rehmsmeier M. RNAhybrid: microRNA target prediction easy, fast and flexible. Nucleic Acids Res 2006; 34:W451-W454.

21 Mathews DH, Disney MD, Childs JL, Schroeder SJ, Zuker M, Turner DH. Incorporating chemical modification constraints into a dynamic programming algorithm for prediction of RNA secondary structure. Proc Natl Acad Sci USA 2004; 101:72877292.

22 Farazi TA, ten Hoeve JJ, Brown M, et al. Identification of distinct miRNA target regulation between breast cancer molecular subtypes using AGO2-PAR-CLIP and patient datasets. Genome Biol 2014; 15:R9.

23 Kong X, Li G, Yuan Y, et al. MicroRNA-7 inhibits epithelial-to-mesenchymal transition and metastasis of breast cancer cells via targeting FAK expression. PLoS One 2012; 7:e41523.

24 Ma L, Young J, Prabhala H, et al. miR-9, a MYC/MYCN-activated microRNA, regulates E-cadherin and cancer metastasis. Nat Cell Biol 2010; 12:247-256.

25 Hiscox S, Jiang WG, Obermeier K, et al. Tamoxifen resistance in MCF7 cells promotes EMT-like behaviour and involves modulation of $\beta$-catenin phosphorylation. Int $J$ Cancer 2006; 118:290-301

26 Raffo D, Berardi DE, Pontiggia O, Todaro L, de Kier Joffe EB, Simian M. Tamoxifen selects for breast cancer cells with mammosphere forming capacity and increased growth rate. Breast Cancer Res Treat 2013; 142:537-548.

27 Jiang Y, Zhao X, Xiao Q, et al. Snail and Slug mediate tamoxifen resistance in breast cancer cells through activation of EGFR-ERK independent of epithelial-mesenchymal transition. $J$ Mol Cell Biol 2014; 6:352-354.

28 Jin K, Kong X, Shah T, et al. The HOXB7 protein renders breast cancer cells resistant to tamoxifen through activation of the EGFR pathway. Proc Natl Acad Sci USA 2012; 109:27362741.

29 Betel D, Wilson M, Gabow A, Marks DS, Sander C. The microRNA.org resource: targets and expression. Nucleic Acids Res 2008; 36:D149-D153. 
30 Lewis BP, Burge CB, Bartel DP. Conserved seed pairing, often flanked by adenosines, indicates that thousands of human genes are microRNA targets. Cell 2005; 120:15-20.

31 Huang da W, Sherman BT, Lempicki RA. Systematic and integrative analysis of large gene lists using DAVID bioinformatics resources. Nat Protoc 2009; 4:44-57.

32 Ashburner M, Ball CA, Blake JA, et al. Gene ontology: tool for the unification of biology. The Gene Ontology Consortium. Nat Genet 2000; 25:25-29.

33 Huang B, Warner M, Gustafsson JA. Estrogen receptors in breast carcinogenesis and endocrine therapy. Mol Cell Endocrinol 2014; 418 Pt 3:240-244..

34 Garcia-Becerra R, Santos N, Diaz L, Camacho J. Mechanisms of resistance to endocrine therapy in breast cancer: focus on signaling pathways, miRNAs and genetically based resistance. Int J Mol Sci 2012; 14:108-145.

35 Scherbakov AM, Andreeva OE, Shatskaya VA, Krasil'nikov MA. The relationships between snaill and estrogen receptor signaling in breast cancer cells. J Cell Biochem 2012; 113:2147-2155.

36 Hardy KM, Booth BW, Hendrix MJ, Salomon DS, Strizzi L. ErbB/EGF signaling and EMT in mammary development and breast cancer. J Mammary Gland Biol Neoplasia 2010; 15:191-199.

37 Cano A, Perez-Moreno MA, Rodrigo I, et al. The transcription factor snail controls epithelial-mesenchymal transitions by repressing E-cadherin expression. Nat Cell Biol 2000; 2:76-83.

38 Li Y, Masaki T, Yamane D, McGivern DR, Lemon SM. Competing and noncompeting activities of miR-122 and the 5 exonuclease Xrn1 in regulation of hepatitis $\mathrm{C}$ virus replication. Proc Natl Acad Sci USA 2013; 110:1881-1886.

39 Winter J, Diederichs S. Argonaute proteins regulate microRNA stability: Increased microRNA abundance by Argonaute proteins is due to microRNA stabilization. RNA Biol 2011; 8:1149-1157.

40 Chatterjee S, Grosshans H. Active turnover modulates mature microRNA activity in Caenorhabditis elegans. Nature 2009; 461:546-549.

41 Brown JA, Bulkley D, Wang J, et al. Structural insights into the stabilization of MALAT1 noncoding RNA by a bipartite triple helix. Nat Struct Mol Biol 2014; 21:633-640.

42 Wilusz JE, JnBaptiste CK, Lu LY, Kuhn CD, Joshua-Tor L, Sharp PA. A triple helix stabilizes the $3^{\prime}$ ends of long noncoding RNAs that lack poly(A) tails. Genes Dev 2012; 26:23922407.

43 Jackson RJ. Cytoplasmic regulation of mRNA function: the importance of the 3' untranslated region. Cell 1993; 74:9-14.

44 Kuersten S, Goodwin EB. The power of the 3' UTR: translational control and development. Nat Rev Genet 2003; 4:626637.

45 Tay Y, Rinn J, Pandolfi PP. The multilayered complexity of ceRNA crosstalk and competition. Nature 2014; 505:344-352.

46 Ebert MS, Sharp PA. Roles for microRNAs in conferring robustness to biological processes. Cell 2012; 149:515-524.

47 Knowlden JM, Hutcheson IR, Jones HE, et al. Elevated levels of epidermal growth factor receptor/c-erbB2 heterodimers mediate an autocrine growth regulatory pathway in tamoxifen-resistant MCF-7 cells. Endocrinology 2003; 144:1032-
1044.

48 Bocker W. [The WHO classification of breast tumours and tumours of the female genital organs: pathology and genetics.] Verh Dtsch Ges Pathol 2002; 86:116-119.

49 Elston CW, Ellis IO. Pathological prognostic factors in breast cancer. I. The value of histological grade in breast cancer: experience from a large study with long-term follow-up. Histopathology 1991; 19:403-410.

50 Willmarth NE, Albertson DG, Ethier SP. Chromosomal instability and lack of cyclin E regulation in hCdc4 mutant human breast cancer cells. Breast Cancer Res 2004; 6:R531-539.

51 Sheridan C, Kishimoto H, Fuchs RK, et al. CD44 $/ \mathrm{CD} 24^{-}$ breast cancer cells exhibit enhanced invasive properties: an early step necessary for metastasis. Breast Cancer Res 2006; 8:R59.

52 Giordano C, Catalano S, Panza S, et al. Farnesoid X receptor inhibits tamoxifen-resistant $\mathrm{MCF}-7$ breast cancer cell growth through downregulation of HER2 expression. Oncogene 2011; 30:4129-4140.

53 Kuo PL, Hsu YL, Cho CY. Plumbagin induces G2-M arrest and autophagy by inhibiting the AKT/mammalian target of rapamycin pathway in breast cancer cells. Mol Cancer Ther 2006; 5:3209-3221.

54 Ortega-Cava CF, Raja SM, Laiq Z, et al. Continuous requirement of ErbB2 kinase activity for loss of cell polarity and lumen formation in a novel ErbB2/Neu-driven murine cell line model of metastatic breast cancer. J Carcinog 2011; 10:29.

55 Mukhina S, Mertani HC, Guo K, Lee KO, Gluckman PD, Lobie PE. Phenotypic conversion of human mammary carcinoma cells by autocrine human growth hormone. Proc Natl Acad Sci USA 2004; 101:15166-15171.

56 Wellner U, Schubert J, Burk UC, et al. The EMT-activator ZEB1 promotes tumorigenicity by repressing stemness-inhibiting microRNAs. Nat Cell Biol 2009; 11:1487-1495.

57 Orom UA, Lund AH. Isolation of microRNA targets using biotinylated synthetic microRNAs. Methods 2007; 43:162-165.

58 Chu C, Qu K, Zhong FL, Artandi SE, Chang HY. Genomic maps of long noncoding RNA occupancy reveal principles of RNA-chromatin interactions. Mol Cell 2011; 44:667-678.

59 Leinonen R, Sugawara H, Shumway M, International Nucleotide Sequence Database Collaboration. The sequence read archive. Nucleic Acids Res 2011; 39:D19-D21.

60 Langmead B, Trapnell C, Pop M, Salzberg SL. Ultrafast and memory-efficient alignment of short DNA sequences to the human genome. Genome Biol 2009; 10:R25.

61 Corcoran DL, Georgiev S, Mukherjee N, et al. PARalyzer: definition of RNA binding sites from PAR-CLIP short-read sequence data. Genome Biol 2011; 12:R79.

(Supplementary information is linked to the online version of the paper on the Cell Research website.)

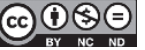

This work is licensed under a Creative Commons Attribution-NonCommercial-NoDerivs 4.0 Unported License. The images or other third party material in this article are included in the article's Creative Commons license, unless indicated otherwise in the credit line; if the material is not included under the Creative Commons license, users will need to obtain permission from the license holder to reproduce the material. To view a copy of this license, visit http://creativecommons.org/licenses/by-nc-nd/4.0/ 